\title{
Structure of the Scientific Theories
}

\author{
Mário J. de Oliveira ${ }^{*}$ \\ ${ }^{1}$ Universidade de São Paulo, Instituto de Física, São Paulo, SP, Brasil.
}

Received on December 08, 2020. Revised on January 08, 2021. Accepted on February 04, 2021.

\begin{abstract}
A scientific theory consists of a symbolic framework containing laws and concepts that are derived by deductive reasoning from fundamental laws and primitive concepts, and complemented by the correspondence or the relation between the symbolic concepts and the real objects. The symbolic framework is understood as a representation of the real, constructed by our minds like a realistic painting. In accordance with this point of view, scientific laws are not discovered but conceived or created by our minds. We show how this approach is organized by rational thinking, which is understood as an innate ability of the mind that emerges between childhood and adolescence. We also examine with some detail the structure of the past scientific theories, pointing out their postulates, or fundamental laws, and the primitive concepts. We will restrict ourselves to some of the physical theories, starting with those that emerged in the Hellenistic period, and then proceed to examine other theories until the end of the seventeenth century.

Keywords: Rational thinking, structure of theories, physical theory, fundamental laws and primary concepts.
\end{abstract}

\section{Introduction}

Scientific knowledge [1-4] is a cultural heritage [5] that has been developed since at least the Hellenistic period, having undergone an excitement and experienced a great thrust in the seventeenth century, called the scientific revolution [6-13. Along the course of time, the scientific knowledge was systematized in scientific theories, examples of which among the physical theories are Euclid's geometry, Ptolemy's astronomy, Newton's mechanics, Maxwell's electromagnetism, Clausius' thermodynamics, Boltzmann's kinetic theory, Gibbs' statistical mechanics, Einstein's relativity, and quantum mechanics. How were these scientific theories achieved? How was scientific knowledge acquired? It is widespread the idea that scientific knowledge is acquired by a method that was developed in the period of the scientific revolution, and called the scientific method. The method consists of a detailed observation of the phenomena, followed by the formulation of a hypothesis by inductive reasoning, which is then tested by careful experiments.

Inductive reasoning is understood as a way of inferring a general proposition from many specific observations. For instance, we may imagine Kepler involved in finding which curve will fit the observation data for the orbit of Mars. He tries several curves until he comes up with an ellipse. Or, we may imagine Galileo, after observing many times the oscillations of lamps suspended by long cords, inferring that the period of oscillation of a simple pendulum is proportional to the square root of its length.

However, if we consider the law of inertia conceived by Galileo, it is difficult to see how it could be a result of

\footnotetext{
${ }^{*}$ Correspondence email address: oliveira@if.usp.br
}

induction as the motion of the inanimate bodies around us indicates otherwise. Of course, he could prepare appropriate experiments to observe inertial motion, as he in fact did it. But in this case, he would already have the idea of inertia, and induction would be useless. Similarly, it is difficult to understand how Copernicus could infer by induction that the earth revolves around the sun if our daily observation indicates the contrary. It is well known that Kepler arrived at the planetary motion laws from the astronomical observations carried out by Brahe. But he did not give any clue of how he reached the third law although he provided in his writings the exact date when this law appeared in his mind. These few examples indicate that the formulation of hypotheses is, in fact, an exercise of one's creativity, which we may call an adventure of the mind 5 . It may emerge in several ways, including in the form of an epiphany as that narrated by Kepler.

No matter how the hypotheses or the fundamental laws are achieved, they are an essential part of a scientific theory and from them other laws are derived. In addition to the laws, a scientific theory includes another category consisting of the concepts in terms of which the laws are expressed. However, the scientific theory is not just an arbitrary set of laws and concepts but has a structure dictated by rational thinking.

Rational thinking, which is an innate ability of the mind, requires that the derivation of a law from another be carried out by deductive reasoning. By this type of reasoning, a logical conclusion follows from a premise, which is certainly true if the premise is true. Rational thinking also requires that deductive reasoning have a point of departure to avoid circular reasoning. This requires that one or more laws should be underivable, 
which are the postulates or the fundamental laws. In an analogous manner, some of the concepts are derived from others but there must be one or more of them that cannot be derivable from the others, as required by rational thinking. These are the primitive concepts, the main examples of which are time and space.

The set of laws and concepts organized by rational thinking constitutes an abstract structure that can be one of the fields of mathematics such as geometry. The connection with the real world is achieved by the introduction of a correspondence between the abstract concepts and the real objects. Any scientific theory has a list of correspondence, sometimes called interpretation. The abstract concepts are thus understood as representations of the real object. It is usual to denote the abstract and the real concepts by the same words. If that is the case, the meaning being used should be determined by the context, when necessary.

It is the purpose of the present paper to describe in details the structure of a scientific theory, pointing out the role of rational thinking and deductive reasoning. In the following, we examine with more detail the structure of some of the past scientific theories, remarking their postulates, or fundamental laws, and the primitive concepts. We will restrict ourselves to the physical theories, starting with those that emerged in the Hellenistic period, and then proceed to examine other theories until the end of the seventeenth century.

The common feature of these theories is that the mathematics employed is geometry. When Galileo spoke of mathematics as the language of nature, he meant geometry, and even Newton used geometry in his Principles. This changed at the end of the seventeenth century when the differential and integral calculus was invented independently by Newton and Leibniz. This new field of mathematics offered a powerful tool for the new scientific theories that appeared from the beginning of the eighteen century and are also worth of a detailed analysis in the light of the point of view regarding scientific theories presented here.

The theories will be presented and analyzed here by using a terminology as close as possible to that of the original text. However, for brevity and clearness, sometimes we will make use of algebra, displayed equations, and modern symbols as long as these recourses do not spoil the original meaning. It should be mentioned for instance that Galileo and Newton in his Principles wrote no equations in the analytical sense that we understand them nowadays.

\section{Structure of Knowledge}

\subsection{Rational thinking}

L'homme n'est qu'un roseau le plus faible de la nature; mais c'est un roseau pensant [14. This is how Pascal described the human condition. Man is not but a reed, the weakest in nature; but it is a thinking reed.
Thinking brings dignity and splendor to man, declared Pascal. Even when we doubt ourselves, said Descartes, we are thinking: je pense donc je suis [15]. The development of natural sciences by Descartes was deeply based on a special type of thinking, rational thinking, and for this reason, Cartesian thinking became synonymous with rational thinking. The thinking faculty of man, praised by Pascal and Descartes, and other abilities of the mind such as consciousness and linguistic faculty emerged in the course of human evolution along with the physical specializations such as the bipedalism, the enlarged brain, and the adaptation for speaking [16].

Rational thinking is innate to each human being and evolves at the last stage of the process of cognitive development occurring from childhood to adolescence [17. According to Piaget, there are four stages of cognitive development [18 20. In the sensorimotor stage, from birth to two years, a child perceives the world through movement and their senses. The preoperational stage consists of two substages. The first, from two to four years of age, is characterized by the use of symbols to represent the world such as toys and drawing. In the second, from four to seven years of age, the child becomes more curious and begin to use a primitive reasoning but is engaged in irreversible thought. In the concrete operational stage, from seven to eleven year of age, the child thinks logically and understand reversibility but is limited to physical objects. In the formal operational stage, beginning at eleven years of age, the child acquires the logical abstract thought, or rational thinking.

Everyone is endowed with rational thinking. Although it can be improved, this ability cannot be taught because in order to learn it from someone we must be provided with rational thinking itself, in which case learning is unnecessary. However, we may characterize it or, equivalently, we may enunciate the rules dictated by rational thinking. Considering that rational thinking is innate so are these rules. Since the rules are innate they appear to us as obvious or self-evident thoughts. One of them is that contradictory propositions cannot both be true. Other rules are those related to deductive reasonings. The most relevant rule is the origination rule of rational thinking stating that a deductive reasoning must have a beginning, an origin. A deductive reasoning is always carried out from a starting point, excluding thus a circular reasoning.

\subsection{Symbolic framework}

According to Craik, when rational thinking is employed in connection with the observation of real events, it leads to discoveries and to the prediction of new events 21. After observing a set of real events, the observer's mind draws some conclusions concerning these events and, based on these conclusions and by a reasoning process some rules are elaborated from which new events are predicted. This reasoning process is carried out by the use of a symbolic model constructed by the mind, a 


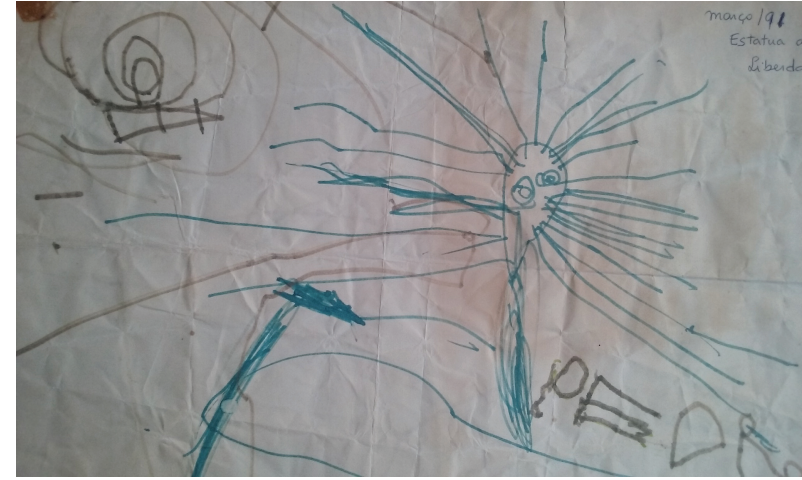

Figure 1: Drawing of the Statue of Liberty by a child of three years old.

mental model, which parallels or is in correspondence with the real events 21 .

The mental model is a symbolic representation of the real world. In this sense, it is similar to the mental framework created by children to represent the physical world around them [17, as proposed by Luquet to explain the children drawings. Luquet argued that children create an internal model, a visual image inscribed on the mind [22]. The children drawing is the imprinting of the internal model in a material medium, and is a realistic representation of a real object, as in the example given in Figure 1. Luquet also considered the prehistoric rock paintings by the primitive men as realistic representations of real objects such as pre-historical animals 23, as shown in Figure 2]

The mental framework, being inscribed in a person's mind, is not accessible to others unless it is printed out. Each one of the drawings shown in Figures 1 and 2 is understood as the imprinting of a mental framework, in this case, a graphic imprinting. We may think of other types of imprinting such as the spoken language, the written language, a small scale model, or any other symbolic framework such as the symbolic language of mathematics. There are various reasons for imprinting in addition to the desire of communicating to others the mental framework. One reason might be a way of recording the mental framework for the purpose of remembering it in the future. Or there might be no reason at all, and the imprinting is just an attempt to imitate nature [16].

A symbolic framework is the imprinting of a mental framework and as such it consists of graphic or written symbols that are real representations of real objects. The mental framework might then be understood as consisting of symbols that are the mental versions of the symbols of the symbolic framework. We may thus assume that the mental and the symbolic framework are two versions of the same framework, the former being internal and not accessible to others, and the latter being the imprinting of the former and accessible to others. Whatever the case, the symbolic framework should not

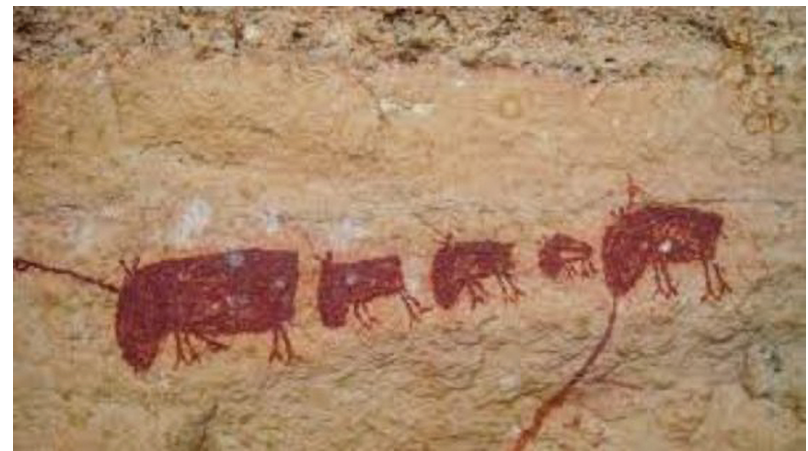

Figure 2: A prehistoric rock painting of a family of capybaras in the Serra da Capivara National Part.

be identified as the real thing. It is the representation of the real but it is not the real, as illustrated by the painting by Magritte, and by the Maxwell equations, shown in Figure 3 .

Figure 4 illustrates the relationship between the thinking abilities, the symbolic framework, and the real world. They form the structure of cognition. We remark again that the symbolic framework is created by the thinking abilities which are not learned but are innate. More clearly, the capacity for creating a framework is innate but the creation of a specific symbolic framework needs external stimuli. In this sense, it is analogous to the acquisition of language by children. The capacity for learning a language is innate but it is necessary external stimuli to acquire a specific spoken language.

\subsection{Scientific theory}

A scientific theory is a symbolic framework created by rational thinking as illustrated on Figure 5 The problem we face now is to state the main features of the symbolic framework which are determined by rational thinking. We distinguish at least two categories of symbols of a scientific theory. One of them consists of the concepts and the other consists of the relations that connect them. A concept may be derived from other concepts but they cannot all be derived concepts otherwise a circular reasoning would take place, in contradiction with the origination rule. Thus some concepts are underivable and are called primitive concepts. The origination rule requires that the relations cannot all be derived from one another. Some relations are underivable, and are called primary relations. The relations that we are referring to are known as laws and the primary relations as postulates, or principles, or fundamental laws.

The symbolic framework ruled by rational thinking can be understood as being constructed by starting from the primitive concepts and primary relations. From them, other concepts and relations can be obtained. New concepts are obtained by defining new ones from the primitive concepts or from the concepts already defined. New relations are derived by using deductive reasoning 


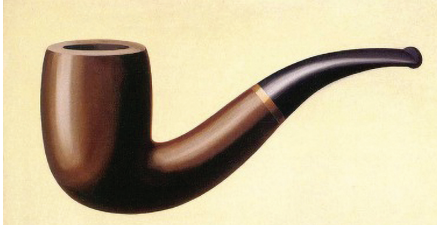

Ceci n'est pas une pipe.

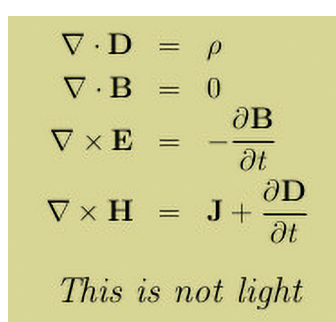

Figure 3: At the left, a painting by Magritte warning: this is not a pipe. Indeed, it is a representation of a pipe. At the right, the Maxwell equations, which are not light but a representation of light.

either from the primary relations or from the relations already derived.

A major example of a symbolic framework constructed in this form, and which we are calling scientific theory, is the Newtonian mechanics, that appeared in the seventeenth century. However, scientific theories emerged before that period 9], a relevant example of which being the Euclidean geometry. Some primitive concepts of Euclidean geometry are points, lines and planes. In Newtonian mechanics, time and space are primitive concepts. The velocity however is a derived concept defined in terms of space and time. In Euclidean geometry, the fifth postulate, which is usually stated in terms of parallel lines, is a primary relation whereas the Pythagorean theorem is a derived relation. In Newtonian mechanics, the second law relating force and acceleration is a primary relation whereas as the conservation of linear momentum is a derived relation.

The features of a scientific theory given above is very similar to those presented by Russo in his analysis of how science was born in the Hellenistic period [9]. According to Russo a scientific theory holds the following features: (a) their propositions are not about real objects but about theoretical ones; (b) its structure is based on deductive reasoning, and consists of a few primary relations; and (c) its application to the real world is based on a correspondence rule. The first two features are in accordance with the properties of the symbolic framework, whereas the third gives the relation of the symbols and the real objects. It is worth mentioning that the representational aspect of a scientific theory was a fundamental aspect of the structure of the physical sciences advanced by Duhem [24 26]. According to him, a physical theory is a system of mathematical propositions, deduced from a small number of principles, which aim to represent as simply, as completely, and as exactly as possible a set of experimental laws [24].

Without the correspondence rule which gives the real counterparts of the theoretical concepts, sometimes called real interpretation or simply interpretation, there is no connection to the real world and a scientific theory is not properly a science but just an abstract theory. However, it is not necessary that all theoretical concepts

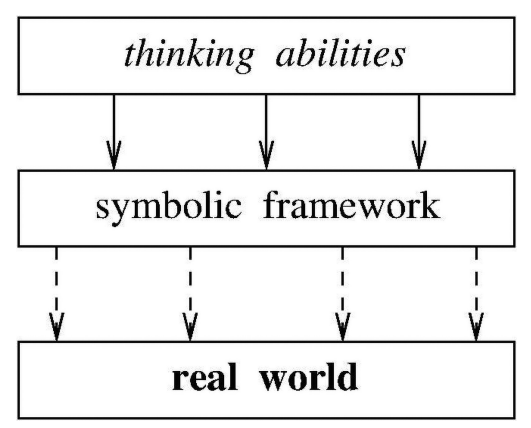

Figure 4: Illustration of the structure of cognition. The thinking abilities are innate structures of the mind. The symbolic framework is created and developed by the thinking abilities and is a representation of the real world.

have real counterparts. This is specially true in the case of quantum mechanics where the concept of wave function has no real correspondence. It is also the case of other theories that sometimes are called non-realistic. One example of this last type is the Ptolemy theory, which includes deferent circles and epicycles that are understood as lacking the real correspondences, but it is nonetheless a genuine scientific theory.

When a real counterpart is a physical quantity such as time or space it is susceptible to be measured. The measurement of a physical quantity requires the definition of a unit of measure and we remark that we should not identify the definition of unit with the definition of the concept itself. This is particularly important in the case of primitive concepts. For instance, we may defined the unit of time as that being given by a simple pendulum with a certain length. However, this is not the definition of time, which is a primitive concept, and undefinable. Another relevant example is that of temperature, which is measured by the thermometer. Although this device gives the unit of temperature, it does not define temperature. In fact, temperature, within the science of thermodynamics is a primitive concept.

The structure of the scientific theory described above gives sense to what is meant by a scientific explanation. If a certain phenomena is ruled by a certain law then the explanation of the phenomena amounts to the explanation of this law which in turn is understood as how this law is derived from the fundamental laws. Therefore, a scientific explanation is the reduction of a law to a more fundamental law, or to the postulates of the theory.

It is worth mentioning that the structure of the scientific theory that we are presenting here, based on postulated and theorems, primitive and derived concepts, it is not an axiomatization or an attempt to axiomatize the theories. The axiomatization will not make a theory more correct of more valid. Of course, consistency is desirable but consistency, whose negation leads to contradiction, is part of our rational thinking 


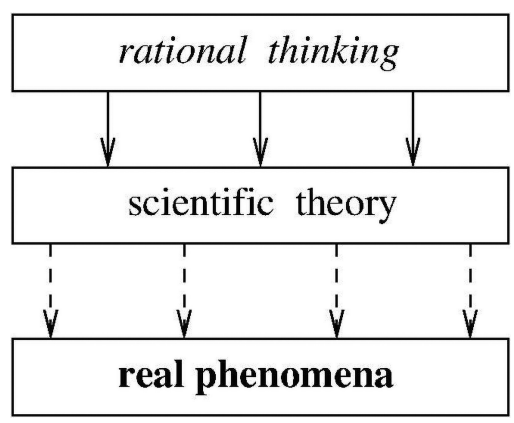

Figure 5: Illustration of the structure of the scientific knowledge. Rational thinking is an innate structure of the mind. The scientific theory is constructed by rational thinking and is a representation of the real phenomena.

and thus the theories are supposed to be conceived with consistency.

An invaluable tool used to reach knowledge is the so-called thought experiment 27 that was used by Einstein in his explanation of the fundamental principles of the relativity theory. Thought experiments were also attributed to Galileo but this view was challenged in the grounds that Galileo in fact performed the experiments [28]. A thought experiment is understood as a statement concerning events taking place in real world without actually performing experiments or making observations. It is a kind of knowledge about the real world called synthetic a priori statement [4. We follow here the logical positivists and deem this type of knowledge as impossible [4. The thought experiment may have unconsciously been impressed on our minds, being in fact based on past experiments and observations, or it might be a form of reasoning based on a mental model created by the mind, and thus understood as a symbolic framework 29]. In fact, it is the expression of a hypothesis in the same sense that the fifth postulate of Euclid is a hypothesis, a postulate, and not a though experiment as one might think at first sight.

\subsection{Laws of nature}

Law in its common sense refers to a practice or a rule of conduct prescribed by an authority or through a general agreement. It is applied to man as a being in full possession of reason and understanding. As it applies to man, who is endowed with free will, a law may or may not be obeyed. A question then arises concerning the use of the term law in scientific language. In the seventeenth century, Boyle stated that nature, being devoid of understanding, cannot follow a prescribed rule, and that he used the word law of nature only for brevity [30]. The argumentation of Boyle seems sound but in the course of time the word law as used in science changed from a prescriptive to a descriptive meaning [30.

The present scientific meaning of law emerged before the middle of the sixteenth century in the fields of optics and astronomy [30. In his work on optics, Roger Bacon in the thirteenth century used the terms laws of reflection, laws of refraction, and in general laws of nature. Later on these terms were used by Kepler in his works in the same subject. In astronomy, Regiomontanus in the fifteenth century used the term law and Copernicus referred to the regular motion of heavenly bodies as laws in the same sense that we use them [30. With Descartes and Newton, the use of law in the modern sense became standard in scientific language.

The descriptive meaning of law fits perfectly well into the structure of scientific knowledge presented here. In accordance with this structure, a scientific law is understood as a theoretical proposition which represents or describes the behavior of a real event. For a better understanding let us consider the Galileo law of falling bodies, which symbolically is given by the equation $x=a t^{2}$, where $x$ is the space, $t$ is the time and $a$ is a constant. This equation belongs to the mathematical structure meaning that $x$ and $t$ are the abstract concepts. It is a law of nature in the sense that if we interpret $x$ and $t$ as the real space and the real time, then if we measure the space at each instance of time, the values found for space will be proportional to those of time squared. This example shows that the term law is used in the descriptive sense and not in the prescriptive sense. In other words, we cannot say that nature prescribes to itself some laws to be obeyed, the collection of which would constitute the real theory.

The scientific laws are theoretical relations that describe the real phenomena. We could not say that the scientific laws are hidden in nature and that they should be discovered or revealed, or that the purpose of science is to search for the real theory hidden in nature. This does not mean that we are denying the existence of the real, but just avoiding to assign to nature something that is a creation of our minds. From these considerations, we conclude that we cannot say that a theory is realistic or more realistic than another, simply because there is no real theory.

If the real theory existed, it would be the perfect and true theory, the theory of everything, the final theory, as nature is unique, and it would make sense to search for such a theory. As it does not exist, the search for it is a search for a chimera. The reduction of one theory to another, understood as deeper and more general, is sometimes pursued because the second is supposed to be closer to the real theory. However, the reduction will not make the theory more valid. The terms model and approximate theory are sometimes used in the place of theory because it is considered that the approach is not yet the supposed real theory.

\section{Euclid}

Euclid lived around the beginning of the third century $\mathrm{BC}$. He is connected to the city of Alexandria and 
must have been acquainted with its great library. He is the author of the mathematical treatise called the Elements concerning mostly with the subject of geometry [31, 32.

The approach to geometry used by Euclid in the Elements is based on the derivation of propositions by deductive reasoning starting from assumptions explicitly stated at the beginning of the treatise, called postulates. This method was invented and developed by the Greeks during the period between six and three hundred years BC, receiving a substantial contribution from the Pythagoreans 33 . Long before this period, some rules of Geometry were already known to the Babylonians and to the Egyptians [31. Some knowledge of the Pythagorean theorem was familiar to the Babylonians. However, their knowledge of geometry was related to the practical tasks demanded by building and land surveying, and the geometric rules they used were taken to be plain truths and not understood as theorems, that is, as propositions to be demonstrated.

The Elements consist of thirteen books containing 465 propositions, obtained by deductive reasoning from five common notions and five postulates. In addition, they contain 131 definitions, including point, line and surface:

A point is that which has no part.

$A$ line is breadthless length.

A surface is that which has length and breadth only.

The definitions of some terms of the Elements as those above are clearly circular, and for this reason these terms should be regarded as primitive 33. However, the choice of which terms should be considered primitive is not unique. It seems natural to consider point, straight line, and plane as included in the set of primitive terms as did Hilbert in his studies on the foundations of geometry 33 .

The common notions and the postulates of the Elements are primary statements, that are not derivable from other statements. The five common notions are understood as universal primary statement and we regard them as part of the structure that we have called rational thinking. The five postulates are geometric primary statement, understood as part of the scientific theory itself. The first postulate states that it is possible to draw a straight line connecting two points and that it is unique. The second is equivalent to say that two straight lines with a common segment are identical. The third states that it is possible to draw a circle with a given point as its center and radius equal to a given rectilinear segment. The fourth asserts that all right angles are equal. The statement of the fifth postulate, illustrated in Figure 6, is: If two straight lines in a plane are intersected by a line segment forming two interior angles on the same side whose sum is less than two right angles then the two lines, if extended indefinitely, will meet each other on the side of the line segment where the angles are less than two right angles. But this postulate is best known in the following simpler and equivalent

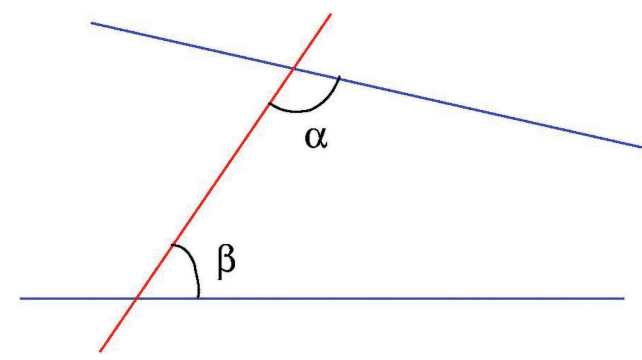

Figure 6: Illustration of Euclid's fifth postulate. If the sum of the angles $\alpha$ and $\beta$ is strictly less than two right angles, then the blue straight lines will meet, if extended indefinitely.

form: Through a given point can be drawn only one line parallel to a given straight line [33].

It is usual to consider Euclidean geometry as pure mathematics but here we are enlarging its meaning and framing it as a scientific theory. This understanding is accomplished by creating a list of correspondence between the theoretical terms and the real objects, which is always possible to do. For instance, a straight line could correspond to a stretched rope or an edge of a rectangular table. In fact, for the ancient Greeks, the geometry was not just a mathematical abstraction but also real although in an idealized form so as to identify a line with a very thin thread [33]. This explains why the category of primitive terms is not distinguished in the Elements 33 . The definition of terms, including the ones that we call primitive, are in fact understood by the Greeks as definitions of real objects such as a line is a breadthless length.

The deductive reasoning used by Euclid is here exemplified by his demonstration of the Pythagorean theorem, which is the proposition 47 of book 1 of the Elements [32]: In right-angled triangles the square on the side subtending the right angle is equal to the squares on the sides containing the right angle. It is understood that the equality here concerns the equality of the areas of the figures. It is a usual practice in dealing with geometry to make no distinction between the geometric figure and its measure. Thus AB may refer to a straight line segment as well as to its length. Although this should be avoided, we will use here the same procedure hoping that the correct meaning can be inferred by the reader from the context.

The demonstration is carried out by Euclid by showing that (a) the area of the rectangle BDLM is equal to that of the square $\mathrm{ABFG}$ and (b) the area of the rectangle CELM is equal to that of the square $\mathrm{ACKH}$, as illustrated in Figure 7 Since the two rectangles make up the square BCED the demonstration is finished. To show the proposition (a) we proceed as follows. The triangles $\mathrm{ABD}$ and $\mathrm{FBC}$ are congruent because the angles $\mathrm{ABD}$ and $\mathrm{FBC}$ are equal and the lengths of $\mathrm{BD}$ and $\mathrm{BC}$ are equal and so are the lengths of $\mathrm{FB}$ and $\mathrm{AB}$. From the congruence it follows that their areas are equal. Now, the area of the triangle BDA is half the area of the rectangle BDLM because they have the same base BD and the 


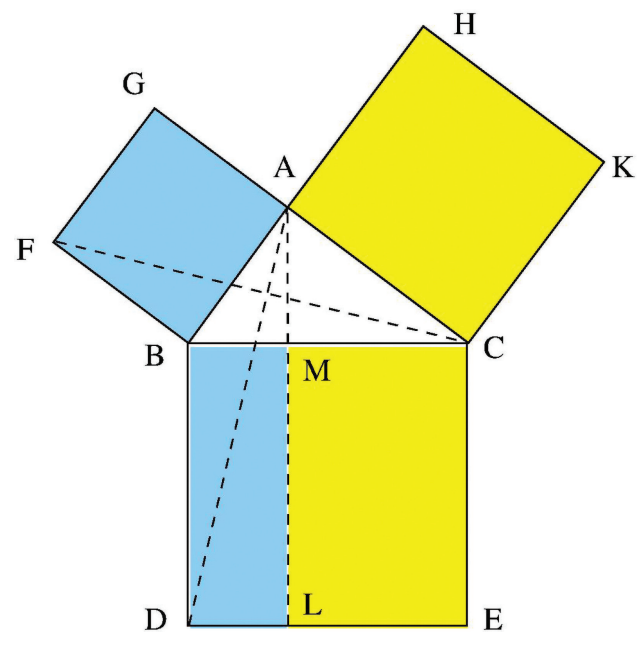

Figure 7: Illustration of proposition 47 of book 1 of the Elements, which is the Pythagorean theorem. The area of the square $B C E D$ is equal to the sum of the areas of the squares $\mathrm{ABFG}$ and $\mathrm{ACKH}$.

same height. Similarly, the area of the triangle FBC is half the area of the square ABFG because they have the same base FB and the same height. Since the triangles $\mathrm{BDA}$ and $\mathrm{FBC}$ have the same area so do the rectangle BDML and the square ABFG, which is proposition (a). The proposition (b) is demonstrated in an analogous manner.

The demonstration that we have just made uses other theorems, not explicitly mentioned, that are derived directly or indirectly from the five postulates. One of them is the proposition 41 of book 1 , which states that the area of a triangle is half the area of a parallelogram of the same base and of the same height. Other familiar propositions of book 1 are as follows. The sum of any two sides of a triangle is greater than the remaining side (proposition 20). The sum of the three interior angles of a triangle is equal to two right angles (proposition 32). Triangles with the same base and same heights have the same area (proposition 37).

In book 2 one finds the geometrical equivalents of certain algebraic identities. Book 3 contains the well know theorems about circles such as the proposition 31: The triangle inscribed in a semicircle is rectangular. The geometry of Euclid is restricted to geometric figures that can be constructed from straight lines and circumferences, which are drawn by a ruler and a compass. Book 4 is devoted to the construction with these Euclidean tools of regular polygons with three, four, five, six, and fifteen sides. In book 5 and 6 , Euclid develops the theory of proportion. The geometric mean is given as proposition 13 of book 6 . The last tree books deals with solid geometry, including the construction of the five regular polyhedra: tetrahedron, octahedron, cube, icosahedron, and dodecahedron. The other books of the Elements deal with number theory and its relation with geometry.

\section{Aristarchus}

Aristarchus is from the island of Samos and it is certain that he was active in $281 \mathrm{BC}$ on account of his observation of the summer solstice of that year [31, 34. He wrote a treatise On the Sizes and Distances of the Sun and Moon, and another also on astronomy, that did not survive, in which he proposed a heliocentric theory. We learn this fact from Archimedes who wrote that Aristarchus formulated the hypothesis that the earth revolves in a circumference around the sun which is also the center of the sphere of the fixed stars [31, 34.

The heliocentric hypothesis is not present in the treatise On the Sizes and Distances, which we are about to examine, possibly because this treatise was written before that on the heliocentric theory. In the treatise On the Sizes and Distances the sun and the moon are assumed to move around circles centered in the earth. The treatise is an extension of geometry and geometric methods of topography to an astronomical scale 9. It contains 6 hypotheses and 18 propositions which are derived from the hypotheses following the same deductive reasoning employed by Euclid in the Elements.

The hypotheses, or postulates, contained in the treatise are as follows 34 :

1. The moon receives its light from the sun.

2. The earth correspond to a point and is the center of the sphere in which the moon moves. That is, the earth can be considered a point when compared to the distance to the moon.

3. When the moon appears to us exactly halved, the great circle which separates its dark and illuminated parts is in direction of our eye.

4. When the moon appears to us exactly halved, its distance from the sun is then less than a quadrant by one thirtieth of a quadrant. That is, $87^{\circ}$.

5. The breadth of the shadow of the earth is that of two moons.

6. The moon subtends one fifteenth part of a sign of the zodiac. One sign of the zodiac encompasses $30^{\circ}$, so that the moon subtends $1 / 15$ of $30^{\circ}$, or $2^{\circ}$.

From the postulates, Aristarchus could derive the following results: The distance of the sun from the earth is greater than 18 and less than 20 times the distance of the moon from the earth. The apparent size of the sun is the same as that of the moon. The diameter of the sun is greater than $19 / 3$ and less than $43 / 6$ the diameter of the earth.

The first result 35] can be derived as follows. From postulate 3 , it follows that when the moon appears exactly in half phase, the earth, moon, and sun are arranged in a right triangle as shown in Figure 8. From this figure we see that the ratio $r$ between the distance sun-earth and the distance moon-earth is the reciprocal of $\sin \alpha$. The value of $\alpha$ given by postulate 4 is 


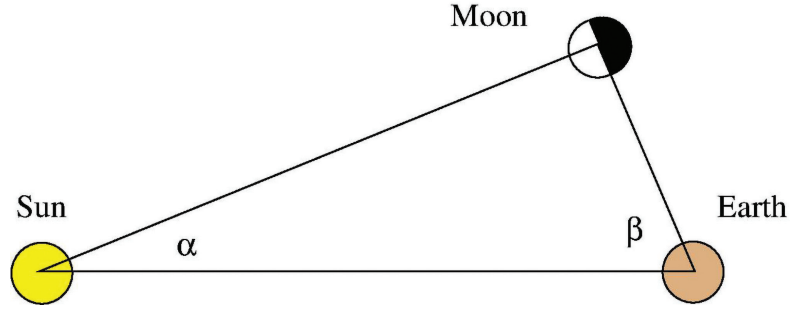

Figure 8: When the moon appears exactly in half phase, the angle earth-moon-sun is $90^{\circ}$. The value used by Aristarchus for the angles $\beta$ is $87^{\circ}$ which gives $\alpha$ equal to $3^{\circ}$. The modern value of $\alpha$ is $10^{\prime}$.

$3^{\circ}$, and the sine of this angle is 0.052 which gives for $r$ the value 19 . However, this was not the reasoning employed by Aristarchus because at that time the trigonometry had not been invented [34]. What he did was to find lower and upper bounds for this quantity.

Aristarchus employed an inequality involving an arc of a circumference and a tangent, which is

$$
\frac{\mathrm{AB}}{\overparen{\mathrm{AB}}}>\frac{\mathrm{AC}}{\overparen{\mathrm{AC}}}, \quad \text { if } \quad \widehat{\mathrm{AB}}>\widehat{\mathrm{AC}} \text {. }
$$

In modern terms, this inequality is equivalent to say that $\tan x / x$ is an increasing function of $x$. Using inequalities of this type and following the Aristarchus reasoning for an arbitrary value of $\alpha$, we find the result $54 / \alpha<r<$ $60 / \alpha$. The derivation of this result involved $\sqrt{2}$ which we replaced by $7 / 5$ as did Aristarchus. Replacing $\alpha$ by $3^{\circ}$, we reach the bounds 18 and 20 found by Aristarchus.

The second result is the proposition 8. Aristarchus states that the same apparent sizes of the sun and of the moon follows from solar eclipse observations. During a solar eclipse, the moon just covers the sun indicating that their apparent discs are equal in size. The hypothesis 6 states that the apparent size of the moon is $2^{\circ}$ and so is that of the sun. This value is to big but Archimedes states that Aristarchus discovered a value for the apparent size of the sun equal to $1 / 720$ of the zodiac, or $30^{\prime} 34$.

The third result concerns the size of the sun, which Aristarchus derived based on the observation that during a lunar eclipse the diameter of the shadow of the earth on the moon is twice the diameter of the moon. The diagram of Figure 9 shows that the rectangular triangles $\mathrm{DAB}$ and $\mathrm{FBC}$ are similar, from which follows that $\mathrm{AD} / \mathrm{BF}=\mathrm{DB} / \mathrm{FC}$. Denoting by $s, e$, and $p$ the radius of the sun, the earth and the dashed circumference shown in Figure 9, we see that $\mathrm{AD}=s-e$ and $\mathrm{BF}=e-p$. But $\mathrm{DB}=\mathrm{SE}$, the distance sun-earth, and $\mathrm{FC}=\mathrm{EM}$, the distance moon-earth, and the ratio $\mathrm{DB} / \mathrm{FC}$ becomes the ratio $\mathrm{SE} / \mathrm{ME}$ which we denote by $r$. Collecting these results we find

$$
\frac{s-e}{e-p}=r
$$

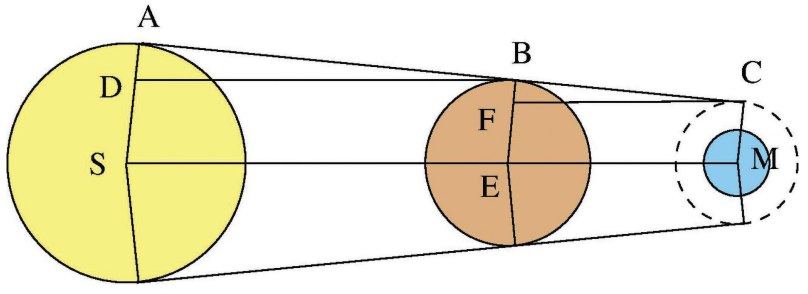

Figure 9: The diagram shows the arrangement of the sun, the earth, and the moon at a lunar eclipse. The points $S, E$, and $M$, which are the centers of the sun, the earth, and the moon, are collinear. The straight line $A B C$ is tangent to the circumferences, and DB and FC are parallel to SEM.

As the apparent sizes of the moon and the sun are the same it follows that their sizes are proportional to their distances to the earth, that is, $s=r m$, where $m$ is the radius of the moon.

Now we need the relation between the radius $p$ of the dashed circumference shown in Figure 9 and the radius of the moon. At first sight it appears that the dashed circle is equal to the disk of the shadow of the earth projected on the moon. Although this is not the case, they are very close. Since from postulate 5 , the size of the shadow of the earth is twice the size of the moon, we may write $p=2 m$. Replacing this result into equation (2) and recalling that $s=r m$, we find the relation

$$
\frac{s}{e}=\frac{r+1}{3} .
$$

Using the values 18 and 20 for $r$ we obtain the bounds $19 / 3$ and 7 for the ratio between the diameters of the sun and the earth. The method of Aristarchus was more complicated and he found the bounds $19 / 3$ and $43 / 6$.

\section{Archimedes}

Archimedes spent most of his life in Syracuse where he died at the age of 75 during the siege of this city, which occurred in $212 \mathrm{BC}$ [36, 37]. He wrote various treatises on several subjects such as geometry, optics, mechanics, statics, and hydrostatics, some of which are known to have been lost. The surviving works include On the Equilibrium of Planes, on the subject of statics, and On Floating Bodies, concerning hydrostatics [36, 37]. The former deals with the equilibrium of flat objects, and was written in two books containing 7 postulates and 25 propositions. The treatise in hydrostatic deals with the equilibrium of bodies in liquids, and consists of two books containing 2 postulates and 19 propositions. The key concept in both treatises is the center of gravity of bodies.

In the two treatises, Archimedes not only employs the same deductive method used by Euclid in the Elements but makes the geometry the underlying theory in both treatises. The main basic concepts related specifically with statics and hydrostatics are weight, equilibrium, 


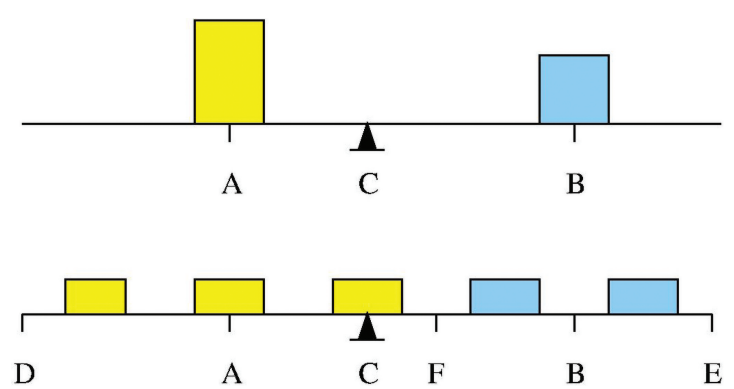

Figure 10: Illustration of the demonstration of the Archimedes law of lever for $n=2$ and $m=3$. The weight of the big yellow block is equal to the three small yellow bricks. The weight of the big blue block is equal to the two small blocks. The small bricks are identical and are equally spaced.

and center of gravity. No explicit definition of these concepts are given. Concerning weight and equilibrium, these terms are used in their meaning in the common language and in fact there is no need for their explicit definitions. We understand them as primitive concepts. As to the third, it is implicit defined by Archimedes as the point at which a body must be suspended to be in indifferent equilibrium [37.

\subsection{Equilibrium of bodies}

The first postulate concerns the equilibrium of bodies as in a beam balance and states that equal weights at equal distances are in equilibrium. From this postulate, one derives at once the proposition 5 of book 1 . If a certain number of bricks of equal weights are distributed along a straight line and are equally spaced, the point of equilibrium is the middle point between the first and the last brick. The equilibrium point is also the center of gravity.

The sixth postulate is essential to demonstrate the law of lever but it is not very clearly formulated. We state this postulate in the following form which will be suitable to demonstrate the law of lever. If a body with its center of gravity at a point $\mathrm{P}$ is in equilibrium with other bodies and is replaced by a set of bodies having the same weight, then the equilibrium is maintained as long as the center of gravity of the set of bodies is placed at the same point $\mathrm{P}$. In propositions 6 and 7 of book 1, Archimedes gives the law of the lever stating that two bodies are in equilibrium at distances reciprocally proportional to their weights.

Following the reasoning of Archimedes, we present the demonstration of the law of the lever for the case of two bodies with the ratio $r$ of weights equal to $n / m$ where $n$ and $m$ are two integers, and $m>n$. The center of gravity of the heavier body is placed at A and the center of gravity of the lighter one at B, as shown in Figure 10 . and they are in equilibrium with $\mathrm{C}$ being their center of gravity. We have to show that the ratio of the distances $\mathrm{AC}$ to $\mathrm{CB}$ is $n / m$. The demonstration is carried out in

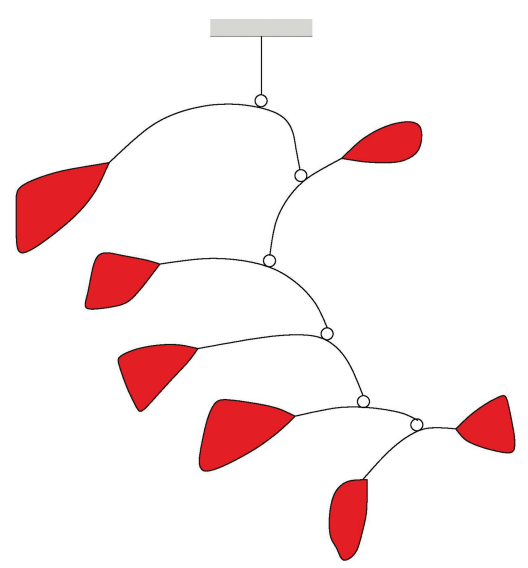

Figure 11: This mobile after Calder illustrates the Archimedes law of the lever. Each small circle is located at the vertical passing through the center of gravity of the mobile attached to the circle. If a mobile is replace by another one with the same weight, the equilibrium is unaltered.

two steps. In the firs step, the body at A is replaced by $m$ bricks with the same weight in such a way that their center of gravity coincides with the point A. In same manner, the body $\mathrm{B}$ is replaced by $n$ bricks with the same weight in such a way that their center of gravity coincides with the point B. By the sixth postulate the equilibrium is not disturbed and the $n+m$ bricks will be in equilibrium.

In the second step of the demonstration, the bricks are arranged is such a way that they are equally spaced, maintaining the two centers of gravity unaltered, so that the equilibrium still takes place by the sixth postulate. Since the bricks all have the same weight and are equally spaced then by the proposition 5 the point of equilibrium is the middle point between the last and first brick. Now $\mathrm{FE}=\mathrm{FB}+\mathrm{BE}$ and $\mathrm{FB}=\mathrm{BE}=\mathrm{AC}$ so that $\mathrm{FE}=2 \mathrm{AC}$, and $\mathrm{DF}=\mathrm{DA}+\mathrm{AF}$ and $\mathrm{DA}=\mathrm{AF}=\mathrm{CB}$ so that $\mathrm{DF}=2 \mathrm{CB}$. Taking into account that $\mathrm{FE}$ and $\mathrm{DF}$ are in the ratio of $n$ to $m$, so are $\mathrm{AC}$ and $\mathrm{CB}$ and this ends the demonstration.

If the ratio $r$ of the weight is not rational, the demonstration is carried out as follows. The two bodies are replaced by two rectangular plates having the same weights. The width of the plates are equal and their lengths are such that they touch each other without superposition. Referring to the Figure 10, the plates have lengths $\mathrm{DF}$ and $\mathrm{FE}$, which are proportional to their weights so that the ratio of $\mathrm{FE}$ and $\mathrm{DF}$ is $r$. We have argued above that this is also the ration between $\mathrm{AC}$ and $\mathrm{CB}$, and this completes the demonstration.

In modern terms we write the law of lever as

$$
p_{1}\left(x_{0}-x_{1}\right)=p_{2}\left(x_{2}-x_{0}\right)
$$

where $p_{1}$ and $p_{2}$ are the weights of the bodies and $x_{1}$, $x_{2}$, and $x_{0}$ are the positions of the bodies and of the equilibrium with respect to a point of reference along an 


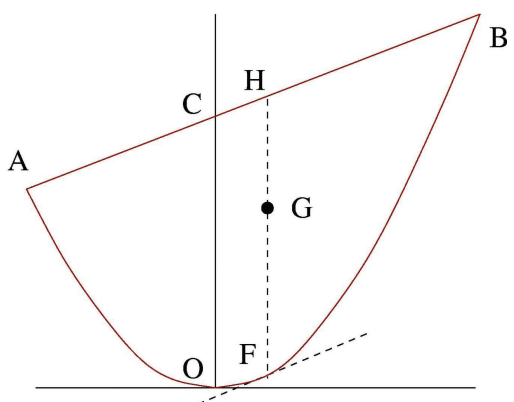

Figure 12: $A$ segment of a parabola $A O B$ with vertex at $O$ and axis $O C$. The inclined dashed line is parallel to $A B$ and tangent to the parabola at $F$. The center of gravity $G$ is on the parallel $\mathrm{FH}$ to the axis at $\mathrm{F}$.

axis. From this equation we find the point of equilibrium,

$$
x_{0}=\frac{p_{1} x_{1}+p_{2} x_{2}}{p_{1}+p_{2}} .
$$

It should be remarked that this equation is not properly the definition of the center of gravity as this quantity is defined as the point at which a body must be suspended to be in indifferent equilibrium. Using the law of the lever we conclude that the center of gravity is given by equation (5). An application of the law of the lever is illustrated in Figure 11

Other propositions of the book 1 deal with the center of gravity of parallelograms and triangles. The location of the center of gravity of these plane figures is determined by the use of the sixth postulate applied to figures of the same area and by the use of the proposition 5 applied to figures of the same area. Proposition 14 states that the center of gravity of a triangle is at the intersection of any two medians.

The aim of book 2 is the determination of the center of gravity of a segment of a parabola, shown in Figure 12 In proposition 8, Archimedes shows that the center of gravity is located at a point $\mathrm{G}$ of the diameter $\mathrm{FH}$ such that $\mathrm{FG}=(3 / 2) \mathrm{GH}$.

\subsection{Hydrostatics}

The treatise in hydrostatics, in two books, has two postulates. The first part of the first postulate is: If contiguous parts of a fluid are on the same level, then the most compressed part pushes away the least compressed part. And the second part is: Each part of the fluid is compressed by the fluid which is right above, except when the fluid is enclosed in something and is under pressure from something else. The second postulate is: Let it be granted that bodies which are forced upwards in a fluid are forced upward along the perpendicular to the surface which passes through their center of gravity.

In book 1, Archimedes derives from the two postulates the main laws of hydrostatics. The principle of flotation is proposition 5: "Any solid lighter than a fluid will, if placed in the fluid, be so far immersed that the weight
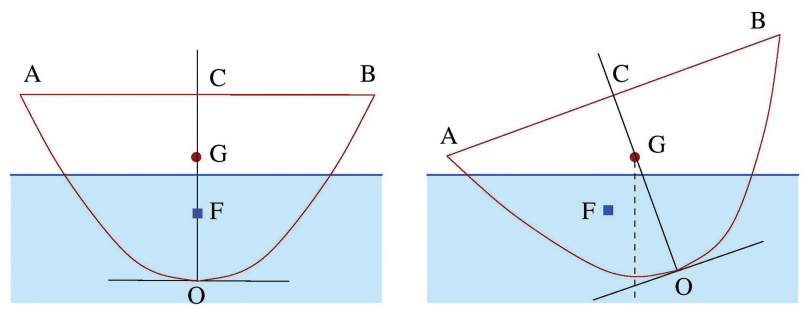

Figure 13: $A C B O$ is a paraboloid of revolution around the axis $O C$, and $G$ is its center of gravity. $F$ is the center of gravity of the part of the paraboloid inside the fluid. In the left panel $F$ and $G$ are in the same vertical, which is the condition for equilibrium. In the right panel the paraboloid is inclined, and $F$ is at the left of the vertical dashed line passing through $G$, an arrangement that restores the equilibrium.

of the solid will be equal to the weight of the fluid displaced." The Archimedes principle is the proposition 6: "If a solid lighter than a fluid be forcibly immersed in it, the solid will be driven upwards by a force equal to the difference between its weight and the weight of the fluid displaced." The statement of proposition 7 is: "A solid heavier than a fluid will, if placed in it, descend to the bottom of the fluid, and the solid will, when wighted in the fluid, be lighter than its true weight by the weight of the fluid displaced." This proposition gives a method of determining the density of a solid in relation to that of the fluid.

In book 2, Archimedes derives the condition for the stability of a floating body. One condition is that the center of gravity $\mathrm{G}$ of the floating body be in the same vertical as the center of gravity $\mathrm{F}$ of the immersed portion, as seen in Figure 13. If they are not in the same vertical the body is not in equilibrium. It is implicit in his writing that Archimedes the equilibrium is restored if $\mathrm{F}$ lies on the opposite site of the vertical passing through $\mathrm{G}$ in relation to the former position of F, as shown in Figure 13

Using these conditions, Archimedes derives the requirements for the equilibrium for a body with the shape of a paraboloid of revolution truncated perpendicular to the axis of revolution. Let as consider such a body with specific gravity less than that of a fluid, placed in a fluid with its axis inclined to the vertical and with the base outside the fluid as shown in Figure 13. Then, if the axis $\mathrm{OC}$ is less or equal $(3 / 4) p$, where $p$ is the principal parameter, the body will not remain in this position but will return to the position where the axis is vertical 36, as can be seen in Figure 13 The principal parameter $p$ is the distance of the focus to the vertex of the parabola. In the remaining of book 2, Archimedes considers the stability for other densities and other values of the ration of the axis and the principal parameter.

\section{Ptolemy}

Ptolemy lived in Alexandria from 100 to 175, approximately, and he has been certainly associated with 
its great library 38. The Almagest 39] is his most important treatise on astronomy, having received this name from the medieval Arabic astronomers. He also wrote treatises on geography, optics and other subjects of astronomy such as the determination of the distances of planets. The Almagest is a systematic exposition of the Greek astronomy which became a standard textbook on astronomy in antiquity and the middle ages [39. Many results contained in this book is due to Hipparchus who lived in the second century $\mathrm{BC} 33$.

The theory presented in the Almagest describes the motion of heavenly bodies without consideration of its causes. The motion is taken for granted and it remains to the theory to describe their trajectories which is carried out by the use of geometry supplemented by the concept of time, understood as a primitive concept.

The Almagest consists of thirteen books written in a logical order. Book 1 contains the hypotheses, which includes a clear statement that a geocentric viewpoint is assumed. The hypotheses or postulates are as follows:

1. The stars move from east to west in circular orbits as if they were pinned in a spherical vault that rotates periodically.

2. The earth is approximately a sphere.

3. The earth is at the center of the spherical starry vault and thus on its axis of rotation.

4. The earth can be considered to be a point when compared with the size of the sphere of the fixed stars.

5. The earth does not have any motion.

Ptolemy assumes that the heavenly bodies are subject to two types of motions. One is the diurnal motion that carries all heavenly bodies from east to west. The fixed stars have only this type of motion, which is identified with the rotation of the celestial sphere. In addition to this motion, the moon, the sun, and the planets have a specific motion, which is the yearly motion.

The location of a body is determined by its distance from the observer and the position of its projection on the celestial sphere centered at the observer, the starry vault. The projection is the apparent position seen by the observer and is determined by the use of a celestial coordinate system consisting of two angles. The astronomy of the Almagest deals mainly with the apparent position and apparent motion on the celestial sphere, and it is usual to omit the word apparent as we will do.

Trigonometry was a basic tool used by Ptolemy. The basic problem addressed by trigonometry is the determination of the length of a chord that is subtended by an given arc of a circumference. Ptolemy used a circumference of radius equal to 60 and arcs measured in degrees. The chord is thus 120 times the sine of half an arc. Ptolemy elaborated a table of chords where we can see that the chord of an arc of one degree is $1+(2 / 60)+(50 / 3600)$. For such a small value, the

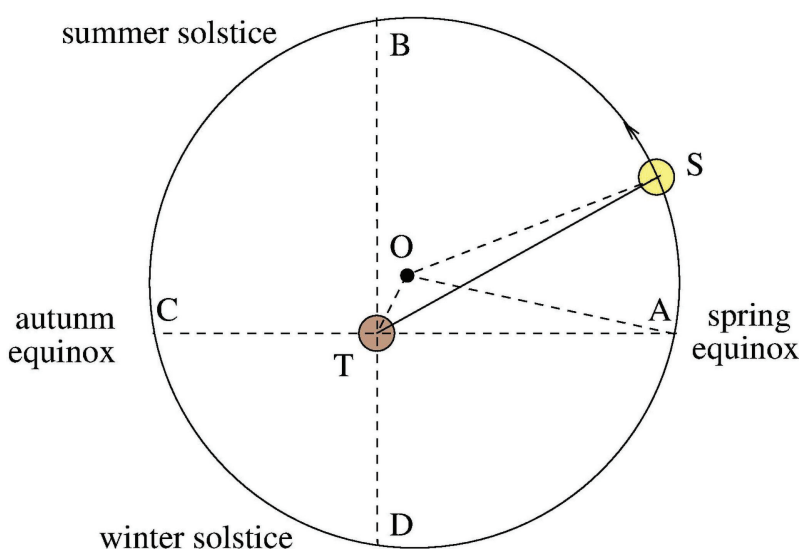

Figure 14: The trajectory of the sun $S$ is the eccentric circumference centered at point $\mathrm{O}$. It moves at constant speed along the circumference which means that the angle SOA increases uniformly. The earth $T$ is off the center. The angle STA, which is the angle an observer sees the sun with respect to $A$, varies non-uniformly.

chord becomes virtually equal to the arc. Since the arc is measured in degrees, it follows that this numerical value is $\pi / 3$, from which we find the value of $\pi$ used by Ptolemy, namely $3+(17 / 120)=377 / 120$ or 3.14167 .

The journey of the sun along its path on the celestial sphere, called ecliptic, is not uniform. It is slight faster in one part of the year and is slight slower in the other part of the year. From the spring equinox to the summer solstice its takes 94.5 days whereas from this last event to the autumnal equinox it takes 92.5 days. Based on these observational data, Hipparchus conceived a model that describes this anomaly motion of the sun, which was used by Ptolemy in book 3. In this model the sun revolves around the earth in an eccentric circular orbit as shown in Figure 14. The speed of the sun along the circular orbit is constant which means that the angle SOA increases uniformly. The earth is off the center. An observer at the earth sees the sun with an angle STA which varies in in an non uniform manner.

As the point $\mathrm{S}$ moves at constant speed along its trajectory, the lengths of the arcs $\mathrm{AB}, \mathrm{BC}, \mathrm{CD}$, and $\mathrm{DA}$ are proportional to their respective travel times, and the same happens to the angles AOB, BOC, COD, and DOA. As the sum of these angles is $360^{\circ}$, the angle $\mathrm{AOB}$ will be equal to $94.5 / 365.25$ times $360^{\circ}$, or $93.14^{\circ}$. Similarly, BOC will be $91.17^{\circ}$. It will be useful to determine the angle OAT. This angle is obtained by subtraction a right angle from half the sum of $\mathrm{AOB}$ and BOC, or $2.155^{\circ}$.

Let us denote by $\theta$ the angle STA, which is the angle that an observer sees the sun in relation to the spring equinox, and by $\phi$ the angle which the straight line OS makes with the direction TA. Then the following relation between can be derived by geometric reasoning

$$
\tan \theta=\frac{\varepsilon \sin \theta_{0}+\sin \phi}{\varepsilon \cos \theta_{0}+\cos \phi}
$$




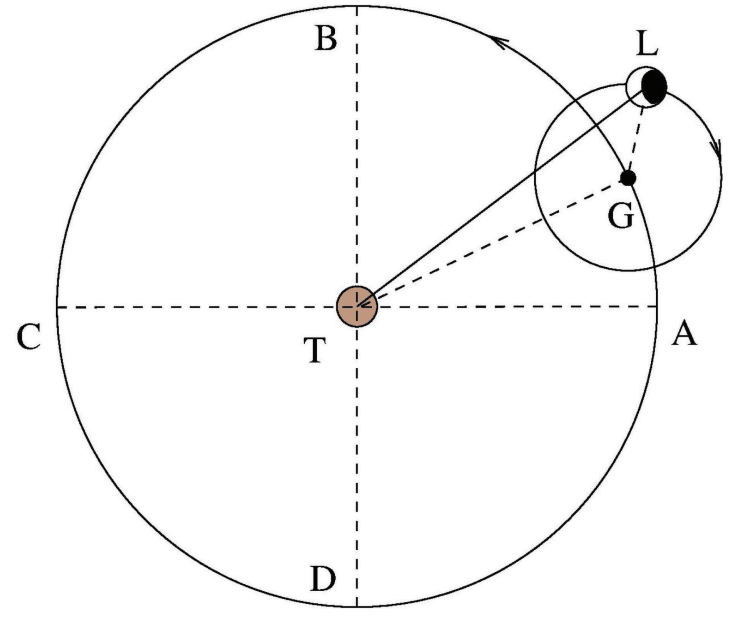

Figure 15: The point $G$ revolves counterclockwise around the earth $\mathrm{T}$ along the great circumference at a constant angular velocity. The moon $\mathrm{L}$ moves clockwise along the small circle around the point $\mathrm{G}$ at a constant angular velocity.

where $\theta_{0}$ is the angle OTA and $\varepsilon$ is the eccentricity, which is the ratio between OT and the radius of the eccentric circumference. These two parameter are found by taking into account that $\theta$ equals one and two right angles when $\phi$, respectively equals $93.14^{\circ}-2.155^{\circ}=90.985^{\circ}$ and $90.985^{\circ}+91.17^{\circ}=182.155$. Replacing these values in equation (6), one finds that the angle ATX equals $65.5^{\circ}$ and the eccentricity is $1 / 24$.

The equation (6) can be understood as the equation of motion, which gives the observation angle $\theta$ in terms of time. It suffices to recall that the point $\mathrm{S}$ moves around $\mathrm{O}$ with an angular velocity $\omega=360 / 365.25$ degrees per day which allows us to write $\phi=\omega t$ where $t$ is the time.

We present now the simplest model considered by Ptolemy for the motion of the moon, which is treated in book 4 of the Almagest. The model was introduce by Hipparchus and consists of two circle, as shown in Figure 15 , the great one being the deferent and the small one the epicycle. The center $\mathrm{O}$ of the deferent revolves counterclockwise around the earth $\mathrm{T}$ at a constant angular velocity $\omega_{1}$ whereas the moon L moves clockwise around $\mathrm{O}$ with a constant angular velocity $\omega_{2}$. Denoting by $\theta$ the angle LTA, by $\phi_{1}$ the angle GTA, and by $\phi_{2}$ the angle that the straight line GL makes with the direction $\mathrm{TA}$, the following relation can be derived by geometric reasoning

$$
\tan \theta=\frac{r \sin \phi_{2}-\sin \phi_{1}}{r \cos \phi_{2}+\cos \phi_{1}},
$$

where $r$ is the ratio between the radius of the epicycle and the radius of the deferent. Bearing in mind that $\phi_{1}$ and $\phi_{2}$ are linear in the time $t$, this equation gives $\theta$ in terms of $t$. The values employed by Ptolemy are $\omega_{1}$ equal to $13^{\circ} 3^{\prime} 54^{\prime \prime}$ per day, $\omega_{2}$ equal to $13^{\circ} 13^{\prime} 46^{\prime \prime}$ per day, and $r$ equal to $5.25 / 60$.

For the planets, Ptolemy conceived a model, shown in Figure 16, which can be understood as modification

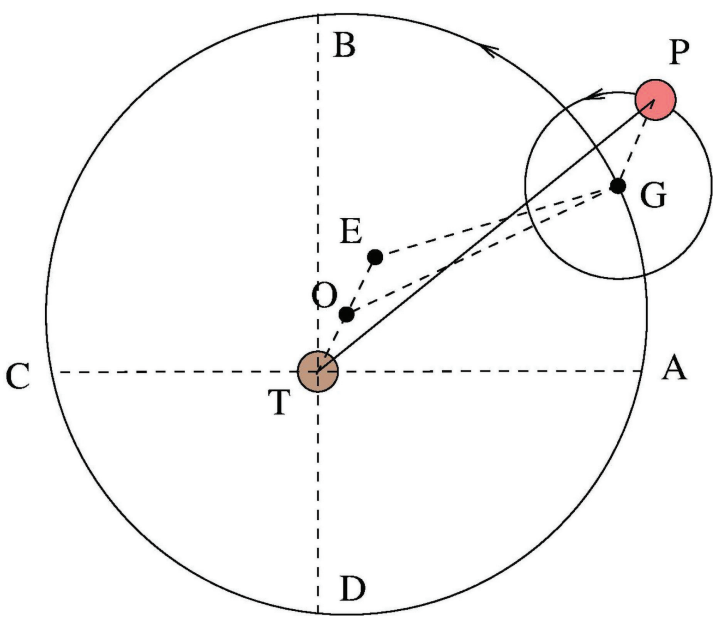

Figure 16: The planet $\mathrm{P}$ orbits counterclockwise along the epicycle centered at the point $G$ which revolves along the deferent circumference centered at $O$. The earth $T$ is off the center and $\mathrm{E}$ is the equant point.

of the deferent-epicycle model. The earth $\mathrm{T}$ is off the center $\mathrm{O}$ of the deferent circle and the center $\mathrm{G}$ of the epicycle moves counterclockwise along the circumference but not at a constant speed. The planet $\mathrm{P}$ revolves counterclockwise around the point $\mathrm{G}$ at a constant angular velocity $\omega_{1}$. The manner in which the point $G$ moves is as follows. Let $\mathrm{E}$ be a point, called equant, located in opposition of the point $\mathrm{T}$, such that $\mathrm{TO}$ equals OE. In the present model, the point $G$ revolves around $\mathrm{E}$ at a constant angular velocity $\omega_{2}$.

Let $\phi_{1}, \phi_{2}$ and $\phi_{3}$ be the angles that the straight lines GP, EG, and OG, respectively, make with the direction TA. Using geometric reasoning, the following equation can be obtained for angle $\theta$ between TP and TA,

$$
\begin{gathered}
\tan \theta=\frac{\varepsilon \sin \theta_{0}+\sin \phi_{3}+r \sin \phi_{1}}{\varepsilon \cos \theta_{0}+\cos \phi_{3}+r \cos \phi_{1}}, \\
\tan \phi_{2}=\frac{\varepsilon \sin \theta_{0}-\sin \phi_{3}}{\varepsilon \cos \theta_{0}-\cos \phi_{3}},
\end{gathered}
$$

where $\theta_{0}$ is the angle OTA, $\varepsilon$ is the eccentricity, the ratio between OT and the radius of the deferent circle, and $r$ is the ratio between the radius of the epicycle and the ratio of the deferent circle. The elimination of $\phi_{3}$ between these two equations give $\theta$ in terms of time as $\phi_{1}$ and $\phi_{2}$ are linear in time.

The model just described is appropriate for the superior planets, Mars, Jupiter and Saturn. Ptolemy assigned to these planets the following values for the eccentricity $\varepsilon$ and for the ratio $r$ of radius of the epicycle and the radius of the deferent:

\begin{tabular}{lcc}
\hline planet & $\epsilon$ & $r$ \\
\hline Mars & 0.100 & 0.658 \\
Jupiter & 0.046 & 0.192 \\
Saturn & 0.057 & 0.108 \\
\hline
\end{tabular}

We have rounded the figures to the third decimal place. 
The model for the inferior planets, Mercury and Venus, is more complicated but includes the epicycle, the deferent circle and the equant point. It suffices to mention here an important additional feature of the model which is the requirement that the center of the epicycle be collinear with the sun 38. This constraint prevents the inferior planets from arbitrarily moving away from the sun.

\section{Ibn al-Haytham}

Hasan Ibn al-Haytham, also known by his Latinized name Alhazen, was born in the last third of the tenth century and lived in Cairo where he died after 1040 [40, 41]. He wrote on many subjects including mathematics, astronomy, medicine, and optics. His major work was in the field of optics, called the Book of Optics [41,44, dealing with visual perception, reflection and refraction of light. He was greatly influenced by the work of Ptolemy on the same subject, and his book had a great influence in the development of optics between the thirteenth and the middle of the seventeenth century [41].

Ibn al-Haytham based his theory of sight in light rays and not in visual rays as did Euclid and Ptolemy. The perception of a visual object is due to light rays coming from the visible object towards the eye. Light is an inherent property of self-luminous bodies and is propagated to other bodies which become also luminous. Each point on the surface of a luminous object is understood as a source of light rays, propagating light in all directions, if transparency permits, through rectilinear trajectories.

The theory is based on geometry so that the principles and concepts of geometry are taken for granted. In addition, there are principles and concepts related to the optics. The most important of these concepts is that of light ray, which we understand as a primitive concept within the theory. The principles concerning the reflection of light are as follows. Let us consider a plane tangent to the surface of reflection of a body at the point of reflection and draw the normal to this point.

1. The line of incidence, the line of reflection and the normal lie in the same plane.

2. The incident line and the reflection line form equal angles to the normal.

3. The image of the object is the point where the extension of the line of reflection intersects the normal dropped from the object at the reflective surface.

These principles are explicit stated in the Optics of Ptolemy [45. The difference lies in the use of light rays by Ibn al-Haytham instead of visual rays.

The principle concerning image location is known as the cathetus rule because the normal to the reflective surface is referred to as the cathetus 46 .

Let us denote the object point by $\mathrm{O}$, the sight point by $\mathrm{S}$, the reflection point by $\mathrm{R}$, and the image point by $\mathrm{I}$,
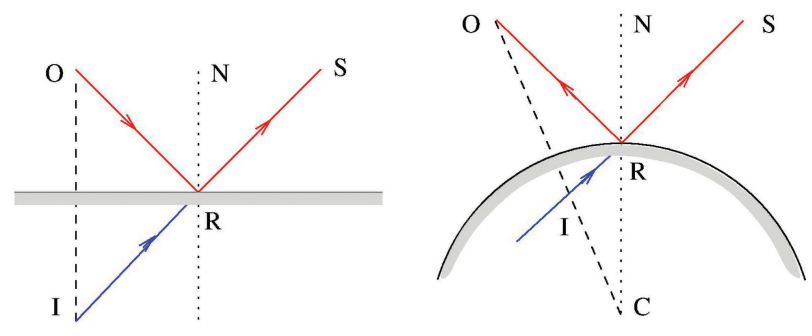

Figure 17: Reflection on a plane and on a spherical mirror. The light ray coming from the object $O$ is reflected at the point $R$ of the reflecting surface and reaches the point $S$. The image $I$ is formed at the point where the cathetus Ol crosses the extension of the reflected ray.

as illustrated in Figure 17. The image I is formed where the cathetus OI crosses the extension RI of the reflected ray $\mathrm{RS}$. In a plane mirror the cathetus is perpendicular to the surface of reflection. In a spherical mirror, it is the line that connects the object to the center of the sphere and thus perpendicular to the reflecting surface. A problem posed by Ibn al-Haytham is the determination of the reflection point, given the object and the sight points. When the mirror is spherical, either convex or concave, this problem is known as the Alhazen problem.

The refraction of light was also treated by Ibn alHaytham in his Book of Optics. The principles of refraction are:

1. The incident ray, the refracted ray and the normal to the interface between the two media lie in the same plane.

2. When a light ray passes from a less to a more dense medium, the angle between the refracted ray and the normal, the refraction angle, is smaller that the angle between the incidence ray and the normal, the incidence angle.

3. The image of the object is the point where the extension of the line of refraction intersects the normal dropped from the object at the reflective surface.

These principles are also explicit stated in the Optics of Ptolemy [45]. The difference lies in the use of light rays by Ibn al-Haytham instead of visual rays.

Let us denote the object point by $\mathrm{O}$, the sight point by $\mathrm{S}$, the refraction point by $\mathrm{R}$, and the image point by $\mathrm{I}$, as illustrated in Figure 18 The image I is formed where the cathetus OI crosses the extension RI of the refracted ray $\mathrm{RS}$. In a plane interface the cathetus is perpendicular to the surface of refraction. In a spherical interface, it is the line that connects the object to the center of the sphere and thus perpendicular to the interface.

Ibn al-Haytham tried to establish quantitative rules concerning the relation between the angle of refraction and the angle of incidence. However, the rules he found are not generally valid and he did not reach the well known sine law of refraction [40, which is usually 

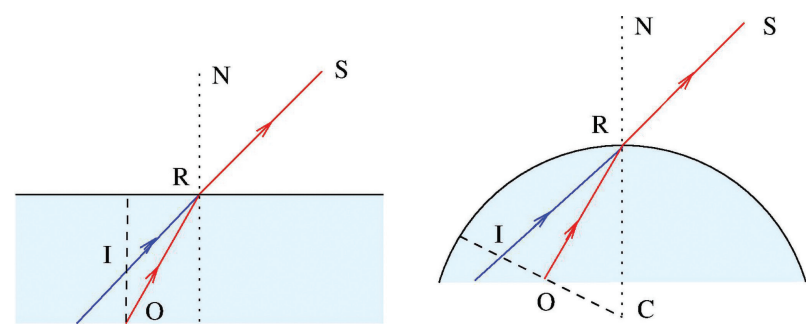

Figure 18: Refraction through a plane and through a spherical interface. The light ray coming from the object $O$ is refracted at the point $R$ of the interface and reaches the point $S$. The image I of the object $\mathrm{O}$ is formed at the point where the cathetus OI crosses the extension of the refracted ray.

written as

$$
\frac{\sin \alpha}{\sin \beta}=n,
$$

where $\alpha$ and $\beta$ are the incidence and refraction angles, respectively, and $n$ is a constant. Ptolemy also failed to reach this law as well as Grosseteste, Witelo, and alFarisi, who were directly influenced by the work of Ibn alHaytham. Kepler also tried to obtain a law of refraction and concluded that the refraction angle is proportional to the incidence angle, which is in accordance with the sine law for small angles [47]. The sine law was eventually stated by Harriot in 1601 and by Snell in 1628, in both cases in unpublished papers, and by Descartes in his Dioptrics published in 1637 [9].

The sine law was in fact discovered much earlier by Ibn Sahl, who described the law in his Book of Burning Instruments written around the year 984, probably in Baghdad [40, 48. Ibn Sahl did not infer the laws from experiments but instead introduced the law as follows. Let $\mathrm{R}$ be the the point of incidence of a ray and let $\mathrm{C}$ and $\mathrm{K}$ be two fixed points on interface between two media such that these three points are collinear as shown in Figure 19. Given the refracted ray RS, it is extends and $\mathrm{B}$ and $\mathrm{A}$ are the points where the extension crosses the normals to the interface at the points $\mathrm{C}$ and $\mathrm{K}$, respectively. Next, the point $\mathrm{O}$ is determined on the normal BC in such a way that RO equals RA, and OR will be the incident ray.

By construction, we see that the ratio $R A / R B$ is equal to the ratio $\mathrm{RK} / \mathrm{RC}$. As the segments $\mathrm{RK}$ and $\mathrm{RC}$ are fixed, it follows that for any refracted ray the ratio $R A / R B$ is constant. But $R A=R O$ and $R O / R B$ is constant, which is the refraction law. It can be written in a more familiar form by writing

$$
\frac{\mathrm{RO}}{\mathrm{RB}}=\frac{\mathrm{CR} / \mathrm{RB}}{\mathrm{CR} / \mathrm{RO}}
$$

and bearing in mind that the numerator is the sine of the angle $\mathrm{CBR}=\mathrm{MRS}$, which is the angle of refraction, and the denominator is the sine of the angle $\mathrm{COR}=\mathrm{ORN}$, which is the angle of incidence.

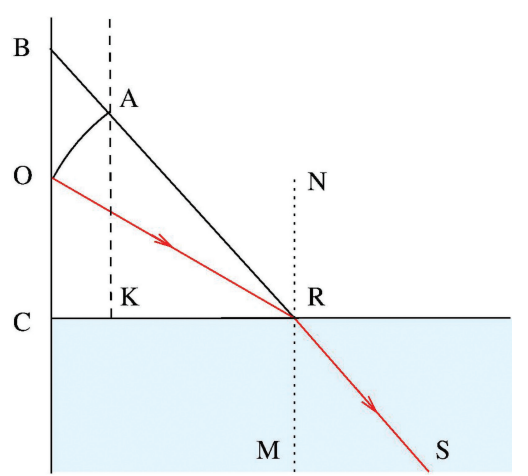

Figure 19: Illustration of the law of refraction as formulated by Ibn Sahl. OR and RS are the incident and the refracted rays and $B R$ is the extension of RS. The points $B$ and $C$ are located at the vertical passing through $O$. The length of $A R$ is equal to OR. The ratio $R A / R B$ is equal to the ratio $R C / R K$.

We have seen above that the image location of an object, either by reflection of by refraction, relies on the cathetus rule, introduced as a principle. The rule remained unchanged until it was challenged by Benedetti in a publication of 1585 and by Kepler in his Optics published in 1605 46. In its place they introduced a method of location based on binocular vision. When an object is directly seen by each of two sight points, it means that two light rays originating from the object reaches each one of these sight points. Thus, the object is located at the point where the rays meet. If the object is seen indirectly, through reflection of refraction, the image is located where the extensions of the two rays reaching the sight points meet. Kepler applied this idea to reflection and refraction. For the case of reflection of plane mirrors and refraction through plane interface, the result is the same as that obtained by the cathetus rule. It should be remarked that the location of the image will depend on the sight point, excepted in the case of plane mirrors.

\section{Copernicus}

Nicolaus Copernicus was born in 1473 at Torun, Poland. He studied at the University of Krakow where he went in 1491 and from 1496 he spent about eight years in Italian universities. From 1504, he lived in two nearby cites in northern Poland. First in Lidzbark until 1510 and then in Frombork until his death in 1543 [38, 49, 50]. In this city he wrote his heliocentric theory called On the Revolutions of the Heavenly Spheres [51, completed around 1532 but published in 1543 just before he died. He formulated his theory much earlier as a brief description of his ideas circulated in manuscript form probably before 1514 [38, 49, 50, 52.

The Copernicus theory is distinct from the Ptolemy theory in placing the sun and not the earth in the center of the world, but it is very similar in other aspects. The fixed stars are pinned in a celestial sphere, which 


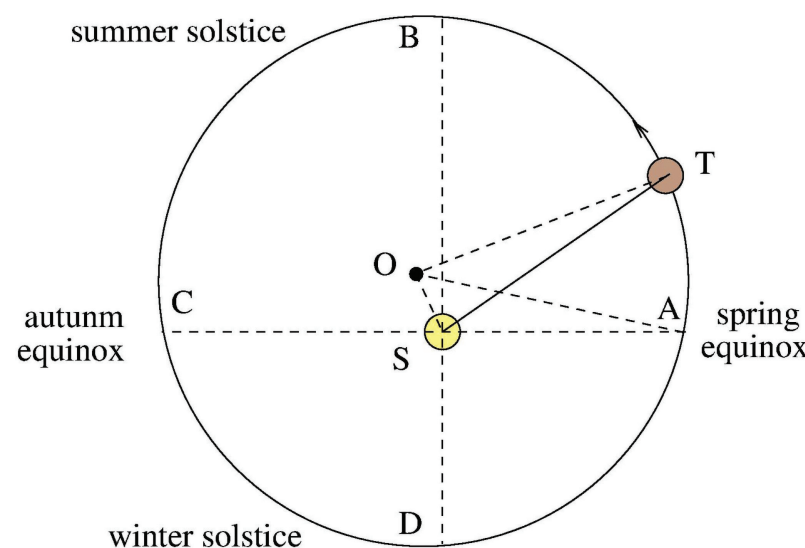

Figure 20: The trajectory of the earth $T$ is the eccentric circumference centered at point $\mathrm{O}$. It moves at constant speed along the circumference which means that the angle TOA increases uniformly. The sun $S$ is off the center and the angle TSA varies non-uniformly.

does not rotate, and the planets, the earth, and the moon follow perfect circles. The daily rotation of the starry vault is apparent and caused by the rotation of the earth around the axis passing from the north and south poles. The motion of the celestial bodies are taken for granted and it only remains to describe geometrically their orbits and positions at each instant of time. Copernicus describes the motion by use of geometrical figures such as deferents, eccentric circles, and epicycles. Like the Ptolemy theory, it is a kinematic theory, describing the motion of heavenly bodies without consideration of its cause.

The principles of the Copernicus theory contained in his Revolutions [51] can be state as follows:

1. The sun is at rest at the center of the immobile sphere of the fixed stars.

2. The earth is spherical and rotates daily from west to east around its fixed poles. This explains the apparent daily motion of the firmament.

3. The size of the earth is insignificant when compared with the size of the heavens.

4. The earth is regarded as one of the planets.

5. The motion of the planets around the sun is uniform, eternal, and circular or compounded of circular motions.

6. The sphere of the fixed stars is the highest. Then follows the spheres of Saturn, Jupiter, Mars, Earth with the Moon, Venus, Mercury, and the Sun at the center of the spheres.

From these principles and the specific models for the planets the aim of the theory is the determination of their positions in relation to the sphere of fixed stars.

One of the first consequences of the theory refers to the position of the earth. If the apparent position of the sun is at a certain sign of the zodiac then the position of the earth will be at the opposite sign. Copernicus gives the

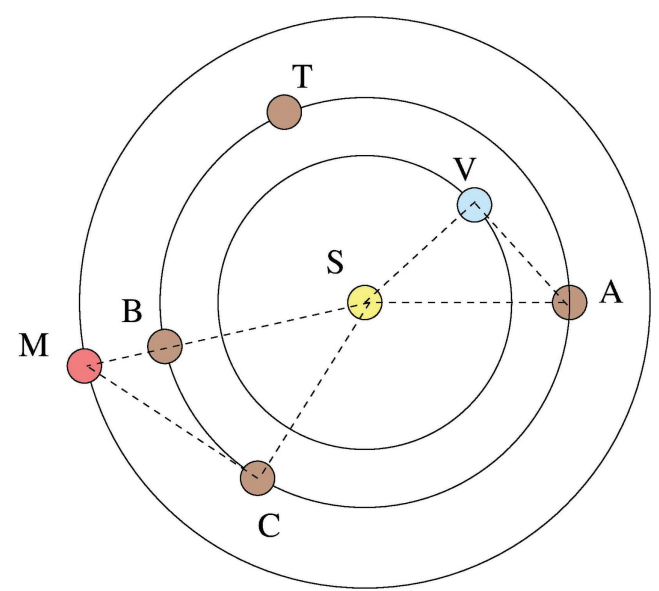

Figure 21: Earth $\mathrm{T}$, Venus $\mathrm{V}$, and Mars $\mathrm{M}$ revolving around the sun $\mathrm{S}$. Venus has an inner orbit whereas Mars has an outer orbit with relation to the earth orbit. A, B, C are three distinct positions of the earth. The position $A$ corresponds to the maximum value of the angle SAV. The position $B$ occurs when Mars is in opposition to the sun, and position $C$ occurs when the angle SCM is equal to a right angle.

following examples: when the earth is passing through the Goat, the sun appears to be in the Crab.

The model for the orbit of the earth around the sun consists of a eccentric circumference as shown in Figure 20. It is similar to that employed by Ptolemy for the orbit of the sun around the earth, but now the roles of the sun and earth are interchanged. The earth $\mathrm{T}$ moves with constant speed along the circumference resulting in a non-uniform variation of the angle AST. Using data coming from his own observations on the motion of the sun, Copernicus finds an eccentricity equal to 0.0323 and the angle OSA equal to $96^{\circ} 40^{\prime}$.

To describe the motion of the moon around the earth, Copernicus adds a small epicycle to epicycle of the Ptolemy model shown in Figure 15. The moon revolves counterclockwise around a certain point which by its turn revolves clockwise around a point moving counterclockwise around the earth. The model for the planets is similar to that of Ptolemy, illustrated in Figure 16, with the earth replaced by the sun.

There are two important consequences that can be drawn concerning the revolution of the planets around the sun. From an observer at the earth, the angle between an inferior planet and the sun cannot be larger than a certain value because these planets have an inner orbit. The maximum elongation is $28^{\circ}$ for Mercury and $46^{\circ}$ for Venus [38. The outer orbits of the superior planets explains their apparent retrograde motion on the starry vault.

Within the Copernicus theory it is possible to determine the distance of a planets from the sun in relation to the distance ST of the earth from the sun 38. Let us consider an inferior planet such as Venus, as shown in Figure 21. If the maximum angle SAV is determined 
then the ratio VS/TS is equal to the sine of SAV. For a superior planet such as Mars, we determine the time it takes for the earth to move from position $\mathrm{B}$ to $\mathrm{C}$. The first position occurs when an opposition of the planet takes place. The second position occurs when the angle of Mars and Sun is equal to a right angle. The arc BC is proportional to time elapsed from $\mathrm{B}$ to $\mathrm{C}$. The ration MS/TS is equal to the inverse of the sine of the angle BSC.

\section{Kepler}

Johannes Kepler was born in 1571 at Weil der Stadt, Germany. He studied from 1589 at the University of Tübingen, where he became acquainted with the Copernicus theory [49, 53. In 1594, he moved to Graz where he lived for six years and then, in 1600, he went to Prague where he became a collaborator to Tycho Brahe. He stayed there until 1612 when he moved to Linz where he lived until 1626. He died two years later. Kepler benefited from the comprehensive works of Tycho Brahe which consisted of the most accurate astronomical observations at the time. These observational data became a star catalog which was published by Kepler in 1627 .

The major astronomical work of Kepler was New Astronomy [54, 55] which contains the areal and the ellipse laws for the planetary motion, published in 1609 while he was in Prague. The harmonic law of planetary motion, concerning the relation between the period and distance of a planet from the sun, appeared in the book Harmonices Mundi [56] published in 1619.

Kepler turned his attention to the theory of Mars around the time he joined Tycho in Prague [53. In the course of the investigation he found it necessary to get more detailed knowledge about the orbit of the earth such as its position at any instant of time. He assumed that the earth moves uniformly on an eccentric circumference around the sun and considered the position of the earth at three times separated by the sidereal period of Mars [57]. In these three instants of time Mars is found in the same position as shown Figure 22 From the astronomical observation of Mars in relation to the sun, the angles of the triangles SAM, SBM, SCM could be found and the three positions, A, B, and C, could be obtained in relation to $\mathrm{S}$ and $\mathrm{M}$.

From the three position of the earth, Kepler determined the radius and the center of the circumference with respect to the sun, and thus the position of the earth on the circumference at any instant of time as the motion was assumed to be uniform. This method yielded the values 0.02401 for the eccentricity of the earth [57. In the following, Kepler realized that the speed of the earth was inversely proportional to its distance from the sun from which he concluded that the line from the earth to the sun sweeps equal area in equal times [53.

Next he turned to the determination of the distances from Mars with the purpose of finding its orbit. He first

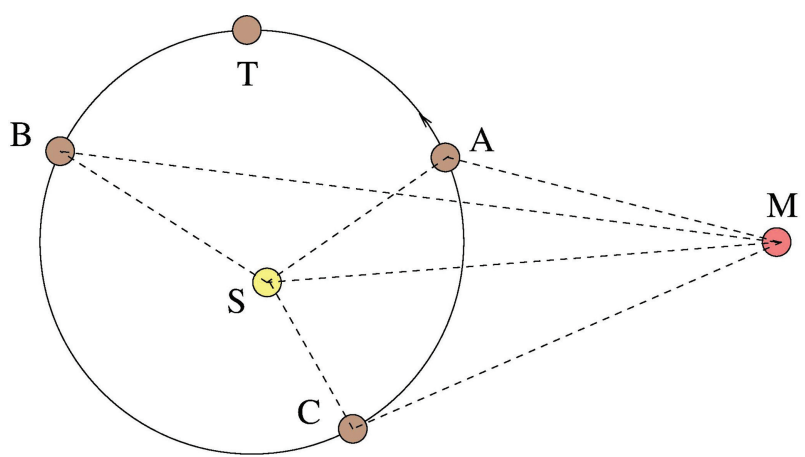

Figure 22: The earth $T$ revolves along the eccentric circumference around the sun $S$. The points $A, B$, and $C$ represents the positions of the earth at three instants such that the mars $M$ is at the same position.

tried to fit the observational data to a circular orbit with an equant point, in accordance with the hypothesis of Ptolemy and Copernicus of assigning circular or compounded circular motions to heavenly bodies. However, he found discrepancies with the distance from Mars obtained from the observational data of Thyco Brahe. The orbit could not be circular but the data showed that the Martian orbit was oval but there was no indication that it should necessarily be an ellipse [57]. Nevertheless, Kepler chose an ellipse to describe the orbit of Mars on account of physical causes, that is, by imagining that a physical cause of motion would result in an elliptical orbit [57].

The statement of the harmonic law by Kepler was as follows: the ratio of the periodic times of any two planets is precisely the ratio of the mean distances raised to the power one and a half [56. Kepler does not give any indication of how he arrived at this law but say that it appeared in his mind on 8 March 1618 [53]. This law as well as the areal and the ellipse laws cannot be considered to be derived from other laws and in this sense it should be understood as basic principles within the Copernicus theory as long as the circular motion principle is replaced by the ellipse law. It should be pointed out that the Kepler laws did not change the kinematics character of the theory. Kepler attempted to find the causes of orbital motion 49 but his planetary laws cannot be understood as a logical derivation from any principle.

\section{Galileo}

Galileo was born in 1564 at Pisa, Italy. In 1581, he was enrolled at the University of Pisa as a student of medicine but left the university in 1585 without a degree. In the following years he taught mathematics at Florence and Siena, and in 1589 he was appointed to the mathematical chair at the university of Pisa, marking the beginning of his scientific career. He stayed in Pisa until 1592 when he was nominated professor 


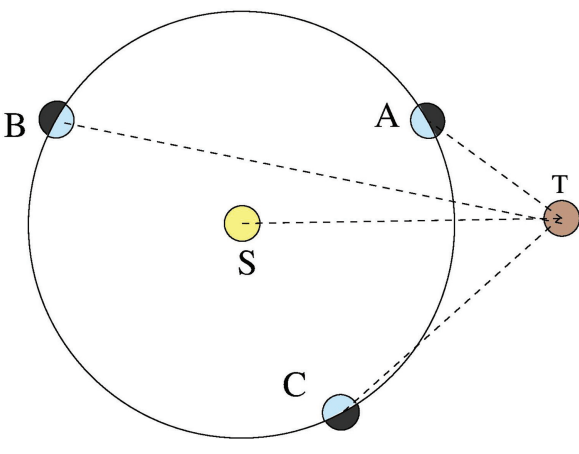

A

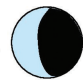

B

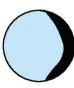

C

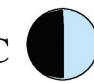

Figure 23: The phases of Venus at three positions $A, B$, and $C$ of the planet with respect to the sun $S$ and the earth $T$. At the right the phases of Venus as seen from the earth at the three positions.

of mathematics at the University of Padua, where he stayed until 1610. Galileo became acquainted with the telescope in 1609 and in the beginning of the following year he discovered the four moons of Jupiter. This great achievement earned him to be called to Florence to be the primary mathematician of the Grand Duke. He moved to Florence in September 1610 and lived there until his death in 1642 [28, 58].

The four moons of Jupiter together with other observations were reported in The Sidereal Message published in 1610 [59, 60. It is worth mentioning that the four moons of Jupiter were also discovered independently by Simon Mayr who gave them their current names: Io, Europa, Ganymede, and Callisto 61. By the end of 1610, Galileo noted that Venus showed phases like the moon, as illustrated in Figure 23. This crucial event confirmed for Galileo the correctness of the Copernicus theory as the Ptolemy theory could not account for this phenomena 28. However, the theory of Tycho Brahe, which places the sun revolving the earth and the planets around the sun, could also account for the phenomena. The rejection by Galileo of the geocentric theory was more properly a result of his commitment to the heliocentric theory [58. If the heavenly bodies are made of the same stuff as that of the earthly bodies and obey the same laws then a large body, the sun, could not revolve around a small one, the earth [28].

The nature of comets was the subject of the Essayer, published in 1623 62. In this book, Galileo criticized the view of one of his opponent that terrestrial parallax was crucial in finding the distance of a comet. Galileo argued that indeed parallax is a genuine research tool, but only if the object seen is proven to be a real physical object. His criticism was methodological as he had no theory concerning the comets [28]. The book discussed not only comets, but other subjects and was essentially a reflection on the methods of scientific inquiry and on the chief role played by mathematics in the investigation of nature [28, 58. He states in the Essayer that the universe è scritto in lingua matematica, e $i$ caratteri son triangoli, cerchi, ed altre figure geometriche, senza $i$ quali mezi è impossibile à intenderne umanamente parola 62 .

The last two treatises written by Galileo were the Dialogue Concerning the Two Chief World Systems, published in 1632 63, and the Discourses and Mathematical Demonstrations Relating to Two New Sciences, published in 1638 64, 65. These two treatises consisted of discussions in the form of a dialogue among three men: Salviati, representing the point of view of Galileo, Simplicio, a follower of Ptolemy and Aristotle, and Sagredo, an enlightened layman. The first treatise was divided into four days and dealt mainly with astronomical topics presenting a defense of the Copernican theory. The second treatise was also divided into four days and treated several topics including suction pumps, weight of air, strength of materials, natural oscillations, and motion of bodies under gravity. In the third day of this treatise, Galileo reaffirms his methods of scientific inquiry stating that principles once established by wellchosen experiments become the foundation of the entire structure.

The study of vibrations appeared much earlier is the Galileo carrier as can be inferred from the following statement: Io ho ben mille volte posto cura alle vibrazioni, in particolare, delle lampade pendenti in alcune chiese da lunghissime corde, inavvertentemente state mosse da alcuno 64. His pendulum law states that the time of oscillations is proportional to the square root of the length of thread. Galileo emphasized that each pendulum vibrates naturally with a determined frequency, which is independent of the weight of the oscillating body 64 .

\subsection{Motion of bodies}

The theory of motion was given in the third and fourth days of the Two New Sciences. Although Galileo is very concerned with the cause of motion, the theory is kinematics. The causes of motion is not included in an explicit form. A relevant feature of the theory is that the laws are independent of the weight of the bodies, a result established by Galileo earlier in his carrier, exemplified by the well known statement that the final velocity of a body in free fall starting from rest does not depend on the weight of the body. It is also implicit that the laws are meant for the motion in which friction is absent or negligible. In the following we will use the language of algebra as a way of expressing the postulates and propositions but we should bear in mind that Galileo expressed them in geometric terms.

Galileo defines two types of motion. The first type is the uniform motion, which means a motion with constant speed. Along a straight line a particle traverses equal distances in equal times. From this last statement it follows that the distance traveled is proportional to the elapsed time. If a particle traverses two distances in equal time then the distances will be in the same ratio as the speeds. Thus the speed one of a body in relation 
to another one is determined by comparing the distances they traveled in a given interval of time. Denoting by $x$ the distance and by $t$ the interval of time, the uniform motion is written as $x=$ ct. The second type is the uniformly accelerated motion. Along a straight line a particle acquires equal increments of speed in equal intervals o time. Denoting by $v$ the velocity then

$$
v=a t
$$

that is, the velocity is proportional to the time elapsed.

Next, Galileo demonstrates the first proposition related to the uniformly accelerated motion. The distance traversed by a body in uniformly accelerated motion, starting from rest, is the same distance traversed by a body in uniform motion with velocity equal to half the final velocity of the accelerated motion. If $v$ is the final velocity, $x$ the distance, and $t$ the interval of time, the first proposition states that

$$
x=\frac{v}{2} t
$$

where $v=a t$. Replacing $v=a t$ into the equation 13 , it follows immediately that

$$
x=\frac{a}{2} t^{2},
$$

which constitutes the second proposition of the uniformly accelerated motion.

The demonstration of the first proposition given by Galileo is as follows. Let us represent by the line AB of Figure 24 the time it takes for a body in uniformly accelerated motion to traverse a certain distance. The final velocity is represented by the line BC. Draw a line parallel to $\mathrm{AB}$ at middle point $\mathrm{F}$ between $\mathrm{B}$ and $\mathrm{C}$, which represent the constant velocity of the uniform motion. It is clear that the rectangle $\mathrm{ABDE}$ and the triangle $\mathrm{ABC}$ have the same area. The demonstration would be finished if the area of the triangle $\mathrm{ABC}$ is shown to be the distance traversed by the body. But Galileo failed to make this cognitive step. Instead, he argues that "what the momenta may lack" in AEF "is made up by the momenta" in FDC, completing the proof. The cognitive step was taken by Huygens who showed that the area of the triangle is identified as the distance traversed by the body [66, 67]. In his treatise on the Pendulum Clock, he showed this result by using upper and lower bounds for the distance traversed as can be seen in Figure 24.

The fundamental law of the theory of motion concerns the free fall of bodies: $A$ body in free fall follows a uniformly accelerated motion. Its immediate consequence is that bodies in free fall follows the law (14). Galileo introduces a second principle, which we call the law of inertia: If a body is in motion in a horizontal plane the body follows a straight line with the velocity unaltered. Using these two principles Galileo demonstrates that the trajectory of a projectile is parabolic.
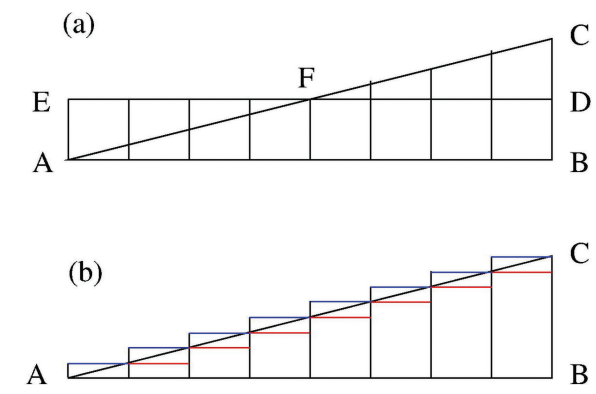

Figure 24: $A B$ represents the time and $B C$ the final velocity of a uniformly accelerated motion. (a) Representation used by Galilleo. (b) Representation used by Huygens.

Galileo assumes that the motion of a projectile can be decomposed in a horizontal motion, which follows the law of inertia, and a vertical motion, that follows the law of free fall. Suppose that a body is moving on an elevated horizontal plane and approaches the edge of the plane. Denoting by $x$ and $y$ the horizontal and vertical distances from the edge, then the motion of the body from the instant it leaves the plane is described by

$$
x=c t, \quad y=\frac{g}{2} t^{2},
$$

because the horizontal motion is uniform and the vertical is uniformly accelerated. From these equations we get the relation between $x$ and $y$,

$$
y=\frac{g}{2 c^{2}} x^{2},
$$

which describes a parabola.

Galileo also considers the oblique launching. This is achieved by using a plane with a certain inclination. He shows that if the angles of inclination differs from $45^{\circ}$ by the same amount, the horizontal distance traveled by the projectile is the same.

\subsection{Motion in inclined planes}

The motion of bodies in inclined planes was a major subject of the studies of Galileo. The laws concerning this subject are derived from the following postulate: The final speeds acquired by bodies descending inclined planes with the same heights are equal. This postulate is equivalent to say that the final velocity is the same as that acquired by the body in a free fall from a height equal to the height of the inclined plane. From this postulate it follows that the motion along the plane is also a uniformly accelerated motion, although Galileo did not seem to demonstrate this result explicitly from the postulate.

If we use the relation $x=(v / 2) t$, where $x$ is the length of the inclined plane and $t$ the time of descending, one concludes that $t$ is proportional to $x$ because the final velocity $v$ is the same for all inclined planes of the same 

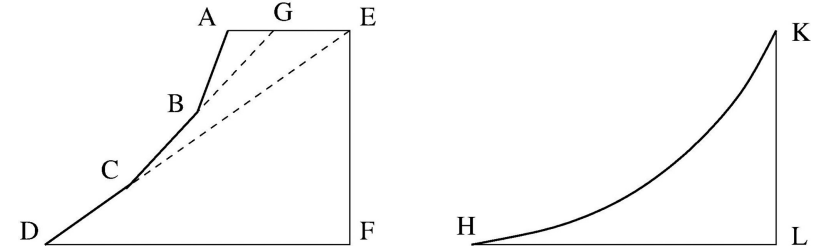

Figure 25: The final velocity of a body starting from rest descending the three inclined planes or any descending curve depends only on the height.

height. This is one of the several propositions derived by Galileo from his principles.

Some of the propositions derived by Galileo were derived later on by Huygens in 1673 in his treatise on the Pendulum Clock [66, 67]. These include the propositions represented by the equations $(12),(13)$ and $(14)$. From results 12 and $(14)$ it follows that

$$
v^{2}=2 a x,
$$

that is, the distance traversed by a body in uniformly accelerated motion, starting from rest, is proportional to the velocity squared, which is proposition 3 of the Pendulum Clock. If the motion does not start from rest but with a velocity $v_{0}$ then the equations $\sqrt{12},(13),(14)$ and (17) are replaced by $v=v_{0}+a t, x=\left(v_{0}+v\right) t / 2$, $x=v_{0} t+(a / 2) t^{2}$, and $v^{2}=v_{0}^{2}+2 a x$.

The distinguish feature of the Huygens approach is his extension of the laws of inclined plane to any descending curve. The final velocity of a body descending any curve is the velocity it acquires by a free fall from a height equal to the vertical distance $h$ from the initial point and the horizontal passing from the final point, that is,

$$
v^{2}=2 g h,
$$

where $g$ is the increase of velocity per unit time in the uniformly accelerated free fall. To show this proposition Huygens consider three inclined planes as shown in Figure 25. From the postulated of the inclined plane, the final velocity at point $B$ starting from $A$ is the same as that start from point G. Starting from G, the final velocity at $\mathrm{C}$ is the same as starting from E. Finally, the final velocity at $\mathrm{D}$ will be the final velocity acquired by the body if it descends the inclined plane ED which turns out to be the velocity of free fall from $\mathrm{E}$ to $\mathrm{F}$. According to the equation (17) this is given by equation (18), which consists of proposition 8 of the Pendulum Clock. This result is immediately generalized by any number of inclined planes and thus to any curve such as that of Figure 25

\section{Descartes}

René Descartes was born in 1596 at La Haye (now Descartes), France. He attended the Jesuit College at
La Flèche most likely from 1607 until 1615. He then went to the University of Poitiers, earning a degree in canon and civil law in 1616, after which he moved to Paris. From 1618 he was in military service in the Netherlands and in Germany. He quit the army in 1620 and spent a few years in France, then stayed in Italy in the period 1623-1625 and went back to France, particularly to Paris. Descartes returned to the Netherlands in 1628 where he lived until 1649. In this year he was invited to the court of Sweden. He died there in 1650 [68, 69].

Descartes wrote treatises on philosophy, mathematics and natural sciences [68, 69. In 1637, he published his Discourse on the Method [15, 70] where he emphasized the use of reason in the development of sciences. This book was accompanied by three essays: Optics, Meteorology and Geometry. This last essay is a mathematical treatise on analytic geometry. The analytic geometry was developed not only by Descartes but also by Fermat, and the idea of representation geometric figures by the use of algebra might have appeared before them 33 .

The basic idea of analytic geometry, stated by Descartes at the very beginning of his Geometry, is that a geometric figure can be represented by straight lines. In geometry the area of a square of sides of length $x$ is equal to $x^{2}$. Thus $x^{2}$ is understood as an area and not as a length. Consider now two similar triangles and let the lengths of two corresponding sides be equal to 1 and $x$, and the lengths of another two corresponding sides be $x$ and $y$. Then considering that the triangles are similar it follows that $1 / x=x / y$ from which we find $y=x^{2}$. Now $x^{2}$ is understood as the length $y$ of a line segment and not as an area. In general any analytic expression involving the length $x$ of a line segment can be understood as the length $y$ of a certain line segment.

An essential feature of analytic geometry is the use of a frame of reference consisting, in the case of plane geometry, of two non parallel straight lines, the axes. Thus each point of a plane figure is represented by two straight lines connecting a point of the curve to the two axes. The analytic relation between the lengths $x$ and $y$ of these two lines, the coordinates, represent the plane figure. As an example, let us consider a parabola, which is geometrically defined as a conic section, the section plane being parallel to a side of the conic surface. Let $x$ be the distance of a point of a parabola to the symmetric axis and $y$ be the distance of this point to the line perpendicular to the symmetric axis at vertex of the parabola. Let $p$ be the length of a certain segment. A geometric property of a parabola is that the area of the square with side $x$ is equal to the area of the rectangle with sides $y$ and $p$. This is a geometric relation between the areas of two plane figures and may be used to define a parabola. Writing this relation in the analytic form $p y=x^{2}$, or yet as $y=x^{2} / p$, it describes a parabola if $x$ and $y$ are interpreted as the coordinates of a frame of reference. 

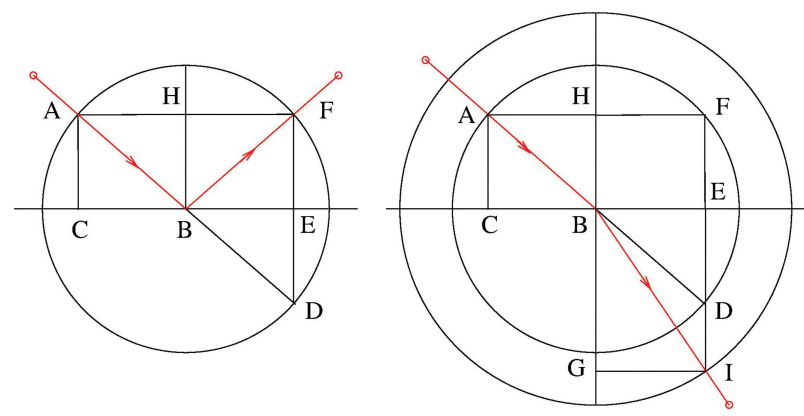

Figure 26: At left, $A B$ and $B F$ are the incident and reflect rays at the point $B$ of the reflecting surface $C B E$. The point $B$ is the center of the circumference. At right, $A B$ and $B I$ are the incident and refracted rays at the point $B$ of the interface $C B E$ of two media. The point $B$ is the center of the two circumferences.

\subsection{Optics and rainbow}

In his essay on Optics [15, 70, Descartes assumes that light is as an action of the medium that is transmitted from one point to the other, stressing that nothing material moves. However, in his reasonings he employs the analogy of light with the motion of a material ball, which means that in fact he uses the emission theory of light. Thus we judge that it is more reasonable to present his theory of reflection and refraction of light contained in his Optics using that light moves from one a luminous body to our eyes with a certain velocity, in agreement with the behavior of a material ball.

The principles of the Descartes optics are as follows. Light is transmitted through straight lines in a transparent homogeneous media. When light hits a plane surface, the transmission is split into two orthogonal transmissions. One of them is perpendicular to the plane surface at the point of incidence. The other is parallel to the plane surface and belongs to the plane of incidence. Reflection and refraction occur in such a way that the parallel transmission remains unchanged.

The laws of reflection are obtained by considering an incident light ray $\mathrm{AB}$ hitting a surface $\mathrm{CBE}$ as shown in Figure 26 $\mathrm{AB}$ is decomposed into two segments $\mathrm{AC}$ and $\mathrm{AH}$, which means that the motion of point $\mathrm{A}$ to $\mathrm{B}$ is decomposed into two projections. One from $\mathrm{A}$ to $\mathrm{H}$, or from $\mathrm{C}$ to $\mathrm{B}$, and the other from $\mathrm{A}$ to $\mathrm{C}$, or from $\mathrm{H}$ to $\mathrm{B}$. In the reflection, the parallel transmission is unchanged, which means that $\mathrm{B}$ goes into $\mathrm{E}$, where $\mathrm{BE}$ is equal to CB. As to the perpendicular component HB, it changes direction in reflection, which means that it changes to $\mathrm{BH}$. The composition of the components $\mathrm{BE}$ and $\mathrm{BH}$ gives the segment $\mathrm{F}$. where $\mathrm{F}$ is on the circumference centered at $\mathrm{B}$ and radius $\mathrm{AB}$. It follows immediately that the angle of incidence $\mathrm{ABH}$ is equal to the angle of reflection $\mathrm{HBF}$.

To find the law of refraction, one proceeds in a similar manner except that now the component HB now changes into the component BG, as shown in Figure 26. To locate the point $\mathrm{G}$, we draw a circumference with center at $\mathrm{B}$

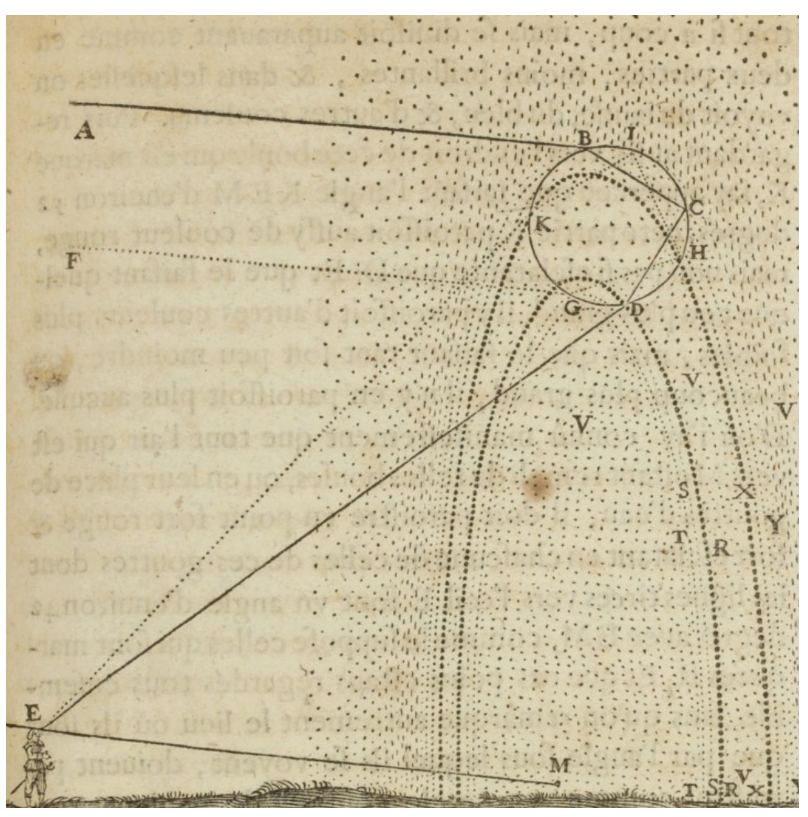

Figure 27: An illustration contained in the Meteorology of Descartes [15] explaining how the rainbow is formed. A light ray coming from the sun suffers an internal reflection in a drop of water and reaches the observer's eye. The principal arc of the rainbow is produced by the ray $A B C D E$, which undergoes a single internal reflection, whereas the secondary arc is produced by the ray FGHIKE, which undergoes two internal reflections.

and a radius $\mathrm{BI}$ such that the ratio $\mathrm{BI} / \mathrm{AB}$ is equal to the ratio of the velocities of light in the two media. From the point $\mathrm{E}$ we draw a vertical line that meet the great circumference at point $\mathrm{I}$, and from this point we draw a line parallel to $\mathrm{BE}$ which meets the point $\mathrm{G}$ in the extension of $\mathrm{BH}$. The components of BI are BE and BG. By construction of the refracted ray $B$, it follows that ratio $\mathrm{BI} / \mathrm{AB}$ is equal to the ratio $\sin \alpha / \sin \beta$, where $\alpha$ is the angle of incidence $\mathrm{ABH}$ and $\beta$ is the angle of refraction $\mathrm{IBG}$. Now, the ratio $\mathrm{BI} / \mathrm{AB}=k$ is the ratio of the radii of the two circumferences which is equal to the ratio of the velocities and thus a constant. We may write than the law of refraction as

$$
\frac{\sin \alpha}{\sin \beta}=n,
$$

where $n$ is the same for all values of the inclination of the incident ray.

A fascinating topic discussed by Descartes was the rainbow, contained in his Meteorology [15, 70. An illustration contained in this treatise is reproduced in Figure 27. Descartes considers a spherical drop of water and how a light ray is reflected inside it. A light ray coming from the sun enters the sphere at point A, suffers an internal reflection in $\mathrm{B}$ and then exit through $\mathrm{C}$, as shown in Figure 28. The angle angle on incidence $\alpha$ at $\mathrm{A}$ is equal to the angle OAP and the angle of refraction $\beta$ is $\mathrm{OAB}$, and they are related by relation 19 . The angle APC is the angle which the rainbow subtends. 


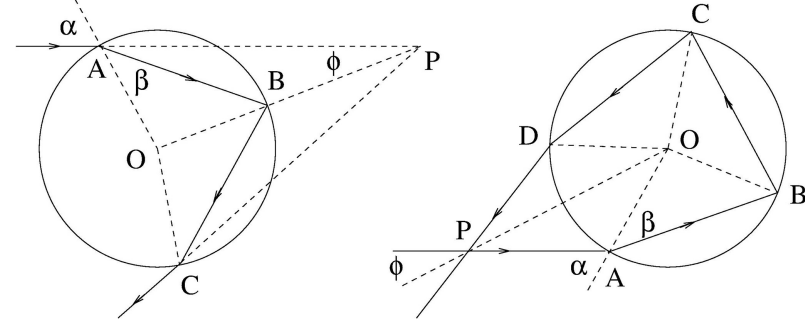

Figure 28: A spherical drop of water. At left, the light ray suffers one internal reflections, giving rise to the principal arc of the rainbow. At right, the light ray suffers two internal reflection, giving rise to the secondary arc of the rainbow.

Defining by $\phi$ half this angle wee see from Figure 28 that $\phi=2 \beta-\alpha$. Given an angle of incidence this relation gives $\phi$ as $\beta$ is related to $\alpha$ by 19 . Descartes chooses the value of $\alpha$ which gives the maximum the value of $\phi$. Carrying out the maximization we find

$$
\sin \phi=\frac{1}{n^{2}}\left(\frac{4-n^{2}}{3}\right)^{3 / 2} .
$$

Replacing $n=250 / 187$ which is the value employed by Descartes we reach the result $2 \phi=41^{\circ} 30^{\prime}$ which is the result found by Descartes.

The result just obtained gives the elevation angle corresponding to the principal arc of the rainbow. To find the elevation angle corresponding to the secondary arc of the rainbow we have to consider two reflections inside the raindrop as shown in Figure 28 . Now the angle $\phi$ is related to $\alpha$ and $\beta$ by $\phi=90^{\circ}+\alpha-3 \beta$. Descartes chooses the minimum value of $\phi$. The minimization of this expression with the condition (19) leads us to the result

$$
\sin \phi=\frac{1}{8 n^{3}}\left(n^{4}+18 n^{2}-27\right) .
$$

Replacing the value $n=250 / 187$, we reach the result given by Descartes, $2 \phi=51^{\circ} 54^{\prime}$.

\section{Pascal}

Pascal was born in 1623 at Clermont, France. In 1631, his father and children moved to Paris. His education began in 1632, receiving lessons from his father himself [71. According to his elder sister, he never went to a school and never had a teacher other than his father [72]. In 1640, the family settled in Rouen. In 1647, Pascal moved to Paris and, except for two long stays in Clermont, he lived in Paris until his death in 1662. Pascal is mainly remembered for his Pensées, concerning the human condition. The students of physical sciences also remember him by his contribution to hydrostatics and this studies of the barometer. He also wrote on philosophy, philosophy of mathematics, and mathematics [71.

In his book New Experiments Concerning the Vacuum [3], published in 1647, Pascal reported experiments that were extensions of those conducted by Torricelli in 1643. Torricelli filled a tube, having one end closed, with mercury and turned it in a vertical position with its mouth inside a vessel with mercury. He observed that the column of mercury reached always the height of "un braccio, e un quarto, e un dito più" 74]. Torricelli explained that we are immersed at the bottom of a sea of air which has a certain height, and that the weight of the air equilibrates the column of mercury [74]. Torricelli concluded that it is not necessary to attribute the explanation of the experiment to a repugnance of nature to vacuum and Pascal drew the same conclusions from his new experiments. In a letter to his brother-inlaw, he explains that it is the weight and pressure of the air that equilibrate the column of mercury [75].

In a response to an opponent concerning these experiments, Pascal reinforces his views concerning the vacuum and set forth his views on the scientific method, emphasizing the use of rational thinking and an approach based on principles or postulates, as did Euclid. He stressed that one also should avoid circular reasoning and circular definition. Pascal gives the following examples of a circular definition, taken from his opponent, that should be avoided: light is the luminairy movement of luminous bodies [72].

In the Account of the Great Experiment on Equilibrium in Liquids of 1648 75, Pascal attached the report from his brother-in-law concerning the experiment that he carried out at the top of Puy de Dôme, near Clermont, in 19 September 1648, at the request of Pascal. He found that the column of the mercury was 23 inches and 2 lignes at the top and 26 inches and 3.5 lignes in the Minims, at Clermont. Pascal himself performed the experiment in the tower of Saint-Jacques, Paris, and found a difference of two lignes between the top and bottom of the tower [75].

The Treatises on the Equilibrium of Liquids and on the Weight of the Mass of Air [76] were completed in 1654 but published in 1663 [71, one year after his death. They are a systematization of the ideas concerning the equilibrium of fluids some of which were similar to those of Stevin, Torricelli, and Mersenne. In these treatises, Pascal presents the concept of pressure and describes several experiments on the equilibrium of fluids, explaining them in the light of his hydrostatic principle.

Pascal states the hydrostatic principle in the first treatise in the following terms: The liquids weigh according to their height. If vessels of distinct shapes, such as those of Figure 29. have the same base, then the force of the liquid on the base will be the same as long as the level of the liquid is the same. We rephrase this statement by saying that the pressure at the bottom is the same, although Pascal did not use the term pressure in the treatises.

If one applies the hydrostatic principle to the machine of Figure 29, we see that it is possible to equilibrate two 

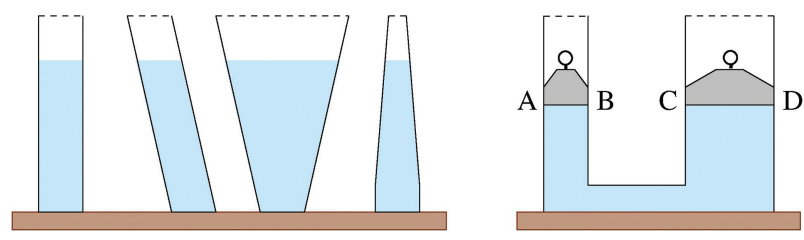

Figure 29: Illustration of the Pascal hydrostatic principle. At left, four vessels with distinct shapes but with the same base. The pressure at the bottom is the same in all the four vessels. At right, the small body equilibrates the large body.

bodies of distinct weights. As the pressure at the level $\mathrm{AB}$ is the same as that of the level CD, then the force will be proportional to the area of the right section of the tube. Then, if two bodies are placed in the two tubes, as shown in Figure 29 then they will be in equilibrium if the ratio of their weights is equal to the ratio of the two areas. We write this result as $p_{1} / p 2=a_{1} / a_{2}$, where $p_{1}$ and $p_{2}$ are the weights, and $a_{1}$ and $a_{2}$ the areas. Suppose that the small body is forced down by a very small distance $b_{1}$. The large body will go up a distance $b_{2}$ such that $a_{1} b_{1}=a_{2} b_{2}$. Replacing this result in the previous equation, one finds

$$
p_{1} b_{1}=p_{2} b_{2} \text {. }
$$

Pascal exemplifies the above result by saying that it is the same thing to make a hundred pounds of water go an inch, as it is to make a pound of water go a hundred inches. He also points out that this relation is the true cause of the equilibrium, and it is similar to the principle used in other machine such as lever machine. Indeed, let us consider the Archimedes law of lever, $p_{1} \ell_{1}=p_{2} \ell_{2}$, where $\ell_{1}$ and $\ell_{2}$ are the lengths of the arms of the lever. If the body weighing $p_{1}$ is pushed down a very small distance $b_{1}$, the body weighing $p_{2}$ will go up a distance $b_{2}$ such that $b_{1} / \ell_{1}=b_{2} / \ell_{2}$, from which we reach equation 22.

Pascal also shows that the two bodies remains in equilibrium if they are perturbed. To this end he uses the principle of center of gravity according to which the equilibrium is lost if the common center of gravity moves downward under a perturbation. He shows that this is not the case of his hydraulic machine. According to Pascal, he demonstrated this principle in a small treatise on mechanics, now lost.

The Archimedes principle is shown by considering that a body immersed in water is under pressure by the water in all sides. But the pressure in the lowest parts of the body is larger that the pressure in the highest parts with the result that the upward force is greater that the downward force. Pascal then concludes that the net force will be that of the water displaced by the body.

In the second treatise, Pascal explains that the weight of the air is the origin of the pressure on every part of a body immersed in it in a way similar to a body immersed in a liquid. He states that the weight of air, which gives rise to the atmospheric pressure, is the origin of all effects which up to his time were attributed to the repugnance of nature to vacuum. The atmospheric pressure explains why it is difficult to open a clogged bellows, or why water flows upwards when a syringe is placed in water and its piston is withdraw. It is also behind the acts of breathing and sucking, particularly, the act of sucking made by a baby in breastfeeding. Pascal also explains that the atmospheric air may vary according to humidity and height.

As the mass of air is limited, the height of the column of liquid in a vertical tube sealed at the top and the mouth immersed in the liquid is also limited. This is the reason why it is impossible to raise water by suction pumps in wells with depths larger than this maximum value. This value is 31 feet for water, two feet three inches and five lignes for mercury, and 34 feet for oil.

It is quite clear that Pascal used an experimental approach in his scientific work. A good example is the experiment carried out by his brother-in-law at the top of the Puy-de-Dôme. Other examples are presented in the treatises on the equilibrium of liquids and the weight of air including the crucial vacuum within a vacuum experiment. In this experiment a Torricelli apparatus is placed inside an evacuated location to show that there is no column of mercury. However, the experiments described by Pascal are not properly devised to infer or to discover a law of nature. They are devised to show the correctness of his fundamental principle or to show that they can be explained by his fundamental principle.

\section{Huygens}

Christiaan Huygens was born in 1629, at the Hague, Netherlands. He was taught at home by a tutor until he was sixteen. In 1645, he went to the University of Leiden and in 1647 to the Orange College at Breda, completing his studies two years later. He moved to Paris in 1666 where he lived for fifteen years. He went back to the Hague in 1681 where he lived until his death in 1695 77.

Huygens carried out researches in astronomy, mechanics, statics, optics, and probability. In astronomy, he discovered in 1655 a moon of Saturn, later called Titan, and resolved the puzzling three spherical form of Saturn found by Galileo. He explained in a publication of 1659 that it was a solid ring orbiting Saturn with an inclination of $20^{\circ}$ to the ecliptic [77.

His treatise on the Pendulum Clock was published in 1673 , dealing with the oscillations of a pendulum and the construction of pendulum clocks. In the second part he is concerned with the cycloidal pendulum which he showed to hold the isochronous property. In the end of this book he published the laws related to the centrifugal forces without giving their derivations. These derivation were contained in a treatise written in 1659 but published only in 1703 [77]. These works on mechanics were based on the theory of Galileo. 
(a)

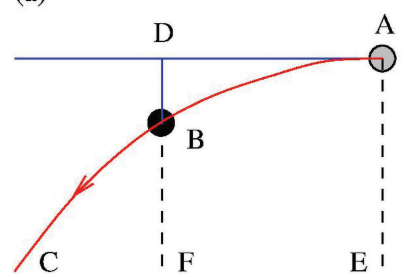

(b)

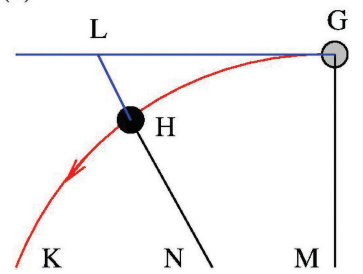

Figure 30: (a) Parabolic trajectory $A B C$ of a projectile $B$ launched horizontally from point $A$. The motion is decomposed into a uniform motion $A D$ and a uniformly accelerated motion DB. (b) Circular trajectory of a body $\mathrm{H}$ tied to a thread $\mathrm{HN}$ over a horizontal plane. The motion is decomposed into a uniform motion GL and a uniformly accelerated motion LH.

It seems that Huygens was led to the development of a wave theory of light to account for the same properties of light that have been considered by Descartes, particularly the sine law of refraction and the independence of light when crossing each other [78. His Treatise on Light published in 1690 [79, 80] but he had written it in 1678 [77]. Huygens considered the speed of light to be finite, a crucial feature in the development of the wave theory. In the introductory part of his treatise he estimated the speed of light to be $162 / 3$ earth diameters per second based on the astronomic observations of Rømer concerning the delays on the eclipses of the moons of Jupiter.

\subsection{Centrifugal force}

The relation between force and velocity in a circular motion was derived by Huygens [81] using an analogy with the motion of a projectile, as illustrated in Figure 30. According to Galileo, the motion of a projectile can be understood as being composed by a horizontal uniform motion and a vertical uniformly accelerated motion. Let us consider the circular motion of a body tied to a thread, as shown in Figure 30. The motion occurs on a horizontal plane so that the weight of the body plays no role. We denote by $r$ the radius of the circumference and by $v$ the velocity of the body.

If the body is released from the thread when it is at the point $\mathrm{G}$, it continues in rectilinear motion with constant velocity. Huygens, thus considers that the circular motion is composed by this rectilinear motion and a uniformly accelerated motion in the direction of the center of the circumference. If after an interval of time $t$ the body is at the point $\mathrm{H}$ then the arc $\mathrm{GH}$ is equal to $v t$. Now, assuming that the arc GH is small compared to $r$, the following geometric relation can be obtained: $\mathrm{LH} / \mathrm{GH}=\mathrm{GH} / 2 r$. In accordance with the assumption made, the cord $\mathrm{GH}$ equals $v t$ and we find $\mathrm{LH}=v^{2} t^{2} / 2 r$, and indeed the component LH corresponds to a uniformly accelerated motion, that is, $\mathrm{LH}=a t^{2} / 2$ where $a=v^{2} / r$.



Figure 31: (a) Simple pendulum: the trajectory $A B C$ is an arc of circumference. (b) Cycloidal pendulum: the trajectory DEF is a cycloid and the period of oscillation is independent of the amplitude.

Huygens argues that the force acting on the body by the thread is proportional to the distance LH and thus proportional to the quantity $a=v^{2} / r$. Next, he gives the following reasoning. In a free fall the acceleration of distinct bodies are the same. However, the force acting on the body is proportional to its gravity or solid quantity. Therefore, the force acting on a body executing a circular motion is proportional to its solid quantity. If we denote this quantity by $m$, the centrifugal force $F$ derived by Huygens can be written in the form

$$
F=m \frac{v^{2}}{r}
$$

although he did not write it in this explicit form.

\subsection{Cycloidal pendulum}

Its seems that Galileo considered the period of a simple pendulum to be independent of the amplitude of swinging. In his treatise on the Pendulum Clock 66, 67], Huygens remarks that the period of a simple pendulum is greater for larger amplitudes and thus not isochronous [77. He shows that, in contrast to a simple pendulum which follows a circular path, a pendulum that follows a cycloidal curve is isochronous, as illustrated in Figure 31. This result was derived by using the Galileo theory of motion, for which he gives a summary of its principles and derive some of its propositions.

A cycloid, shown in Figure 32 is a curve traced by a point at the edge of a circle rolling without sliding along a straight line. Defining $\theta$ as the angle OCP, a point A of the cycloid is given parametrically by

$$
\begin{aligned}
& x=r(\theta-\sin \theta), \\
& y=r(1-\cos \theta),
\end{aligned}
$$

where $x$ is the horizontal distance HA and $y$ is the vertical distance $\mathrm{KA}$, and $r$ is the radius $\mathrm{CO}$ of the circumference which generates de cycloid. A body is released from rest at the point $\mathrm{G}$ defined by $x_{0}=$ $r\left(\theta_{0}-\sin \theta_{0}\right)$ and $y_{0}=r\left(1-\cos \theta_{0}\right)$ where $\theta_{0}$ is the angle OCD. The problem is the determination it takes to reach the lowest point $\mathrm{F}$ of the cycloid 82 . 


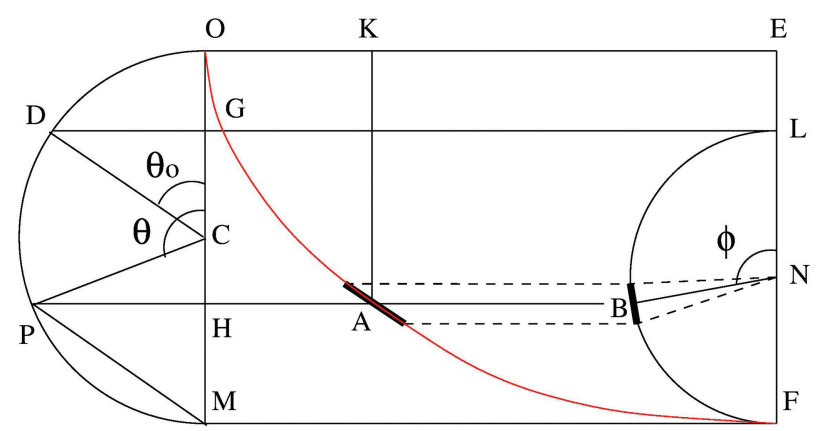

Figure 32: The curve OAF is a cycloid. A body released from rest at any point of the trajectory, such as the point $G$, will take the same time to reach the lowest point $F$. Any point $A$ of the cycloid OAF is mapped onto a point $\mathrm{P}$ of the semicircumference OPM. Any point of the trajectory GAF is mapped onto the small semicircumference LBF.

As the vertical distance traversed by the body is $y-y_{0}$, then according to 18 , the velocity at the point $\mathrm{A}$ is

$$
v=\sqrt{2 g\left(y-y_{0}\right)} \text {. }
$$

The time it takes for the body to travel the distance $s$ of the small inclined segment tangent to the cycloid at A is $\tau=s / v$. Let $\ell$ be the length $\ell$ of the small segment tangent to the circumference LBF at the point B. Next, we wish to determine the relation between $\ell$ and $s$, which is accomplished by finding their slopes. A property of the cycloid states that a tangent of the cycloid at $\mathrm{A}$ is parallel to the line PM. From this property the slope of the small segment at $\mathrm{A}$ is equal to two right angles minus $\theta / 2$. Let us denote by $\phi$ the angle BNL. Then the slope of the small segment at $\mathrm{B}$ is equal to two right angles minus $\phi$. From these results we find

$$
s \cos \frac{\theta}{2}=\ell \sin \phi .
$$

The relation between the angles $\phi$ and $\theta$ is determined as follows. Let us denote by $a$ the radius of the small circumference. As the diameter LF is equal to GM, we find $2 a=r\left(1+\cos \theta_{0}\right)$ or

$$
a=r \cos ^{2} \frac{\theta_{0}}{2} .
$$

The segment HM is $2 r-y=r(1+\cos \theta)=2 r \cos ^{2} \theta / 2$ and equal to $a(1+\cos \phi)=2 a \cos ^{2} \phi / 2$. Therefore,

$$
r \cos ^{2} \frac{\theta}{2}=a \cos ^{2} \frac{\phi}{2},
$$

which is the desired relation between $\theta$ and $\phi$. It can be written as

$$
\cos \frac{\theta}{2}=\cos \frac{\theta_{0}}{2} \cos \frac{\phi}{2} .
$$

Replacing the result (30) in the expression (26), we get $v$ in terms of $\theta_{0}$ and $\phi$,

$$
v=2 \sqrt{g r} \cos \frac{\theta_{0}}{2} \sin \frac{\phi}{2} .
$$

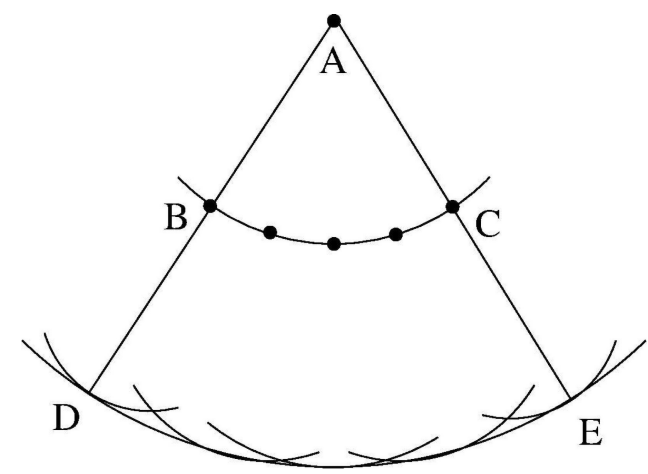

Figure 33: Illustration of the Huygens principle. $B C$ and DE are wave fronts originating from the point $A$, whereas the small arcs are secondary front waves originating from the points on the arc $\mathrm{BC}$.

Collecting the results above we find the following expression for $\tau=s / v$,

$$
\tau=\frac{\ell}{\sqrt{g r}\left(\cos ^{2} \theta_{0} / 2\right)}=\frac{\ell}{a} \sqrt{\frac{r}{g}} .
$$

The time of descent is obtained by summing all segments $\ell$ when $\theta$ varies from $\theta_{0}$ until two right angles, or when $\phi$ varies from zero until two right angle. The sum of these segments is the length of a semi-circumference or radius $a$, that is, $\pi a$. Thus the time of descent is $\pi \sqrt{r / g}$. The period $T$ of a pendulum is four times the time of descent, or

$$
T=2 \pi \sqrt{\frac{4 r}{g}},
$$

and is independent of $\theta_{0}$, and thus independent of the amplitude of oscillations.

\subsection{Wave theory of light}

Huygens introduces the concept of light waves in analogy with sound propagation. Sound spreads through the air from its origin in successive growing spherical surfaces. In same manner, light spreads by spherical surfaces called waves in resemblance to waves produced in water when a stone is thrown in it. However, sound and light are very distinct in respect to underlying media supporting their spreading. The sound that reaches our years is transmitted through the air whereas the light that reaches our eyes is conveye by a matter called ethereal by Huygens. It pervades places devoid of air, such as the Torricelli vacuum, allowing light to traverse it.

Huygens postulates that every particle of a luminous body is a center of spreading of a spherical light wave. He also postulates that each point of a spherical front can be regarded as the origin of a secondary wave, as can be seen in Figure 33 These postulates constitutes the fundamental principle upon which is based his wave theory. 


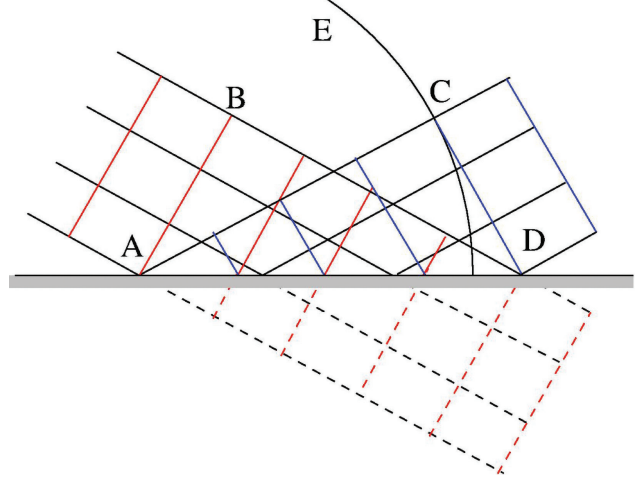

Figure 34: Reflection of light waves. $A B$ is an incident front wave and $C D$ is the reflection front wave. $C E$ is an arc of the circumference with center at $A$ and radius $A C$ equal to $B D$ and understood as a secondary wave front with origin at $A$.

The reflection of a wave light is constructed as follows. Let $\mathrm{AB}$ be a wave front of an incident light wave and $\mathrm{CD}$ that of a reflected light wave as shown in Figure 34. The points $\mathrm{C}$ and $\mathrm{D}$ are understood as belonging to the secondary front waves having their origins at the points $\mathrm{A}$ and B, respectively. Since the front waves have the same radius it follows that $\mathrm{BD}$ equals AC. From this result we conclude that the angle $\mathrm{BAD}$ equals the angle ADC. But these two angles are identified as the angle of incidence $\alpha$ and and angle of reflection $\beta$, respectively, and the law of reflection, $\alpha=\beta$, becomes demonstrated.

The sine law of refraction is derive as follows. Let $\mathrm{AB}$ be a wave front of an incident light wave and $\mathrm{CD}$ that of a refracted light wave, as shown in Figure 35 The points $\mathrm{C}$ and $\mathrm{D}$ are understood as belonging to the secondary front waves having their origins at the points $\mathrm{A}$ and $\mathrm{B}$, respectively. As the rectangular triangles $\mathrm{ABD}$ and $\mathrm{ACD}$ have a common hypotenuse, and bearing in mind that $\mathrm{BAD}$ is identified as the angle of incidence $\alpha$ and ADC is identified as the angle of refraction, we conclude that $\sin \alpha / \sin \beta=B D / A C$. Now, the radius AC of the first wave front is distinct from the radius $\mathrm{BD}$ of the second wave front because because they are proportional to the speed of light in the medium. Denoting by $v_{1}$ and $v_{2}$ the speeds in the upper and lower media, then $B D / A C=$ $v_{1} / v_{2}$ and $\sin \alpha / \sin \beta=v_{1} / v_{2}$, which is the sine law of refraction.

After reaching the refraction law, Huygens showed how his principle could lead to the Fermat principle of least time. Fermat postulated that among all the paths connecting two given points, the actual path taken by a light ray is that with least time. In a correspondence of 1662 , he demonstrated that the sine law of refraction was a consequence of his principle [78]. Huygens demonstrated the reverse, that the least time principle was a result of the sine law, as follows. Let $\mathrm{A}$ and $\mathrm{C}$ two points on distinct media and $\mathrm{ABC}$ a light ray connected them, as shown in Figure 36 Let AFC another path connecting these two points. The line OF

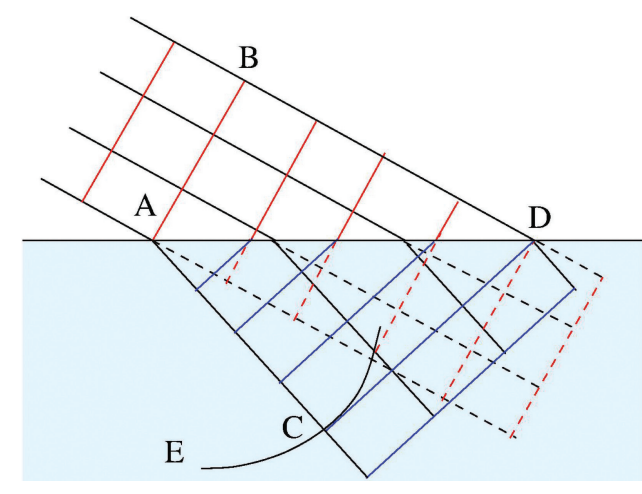

Figure 35: Refraction of light waves. $A B$ is an incident front wave and $C D$ is the refraction front wave. $C E$ is an arc of the circumference centered at $A$ and radius equal to $A C$ which is equal to $B D$ times the ratio between the wave velocities of the lower and upper media. CE is understood as a secondary wave front with origin at $A$.

is parallel to $\mathrm{AB}, \mathrm{AO}$ and $\mathrm{BH}$ are perpendicular to $\mathrm{AB}$, and $\mathrm{GF}$ is perpendicular to $\mathrm{BC}$.

As the angle BFH and BFG can be identified as the angle of incidence and refraction, respectively, then from the law of sines, the ratio HF/BG is equal to the ratio of the speeds of lights on the two media and consequently HG is traversed in the same time as BG. Therefore, the time it takes to travel along $A B G$ is the same as that of OF. But the time to travel AF is larger than that of $\mathrm{OF}$ and thus larger than that of ABG. In addition, the time to travel FC is larger than that of GC, which completes the demonstration. A similar demonstration can be carried out if the point $\mathrm{F}$ is chosen to be at the left of the point B.

Huygens discusses also the refraction of light in the atmospheric air. He argues that as the air is not homogeneous the light ray does not travel in straight lines. A light ray may be understood as traversing a sequence of layers with distinct refractions yielding a non rectilinear path to the light ray. He gives the examples of the sun as it sets in the horizon. The refraction near the horizon makes the sun appear higher than the actual position.

A large part of the treatise is devoted to the discussion of the double refraction of Iceland spar, the transparent form of calcite. To explain this phenomena, Huygens considered that the front wave impinging on the surface of calcite is split into two waves generating the ordinary and extraordinary rays. The ordinary ray is transmitted by spherical waves. The extraordinary ray is transmitted by ellipsoidal waves having different velocities in distinct directions.

\section{Newton}

Isaac Newton was born in 25 December 1642 (Julian calendar) at Woolsthorpe, England. He attended the 


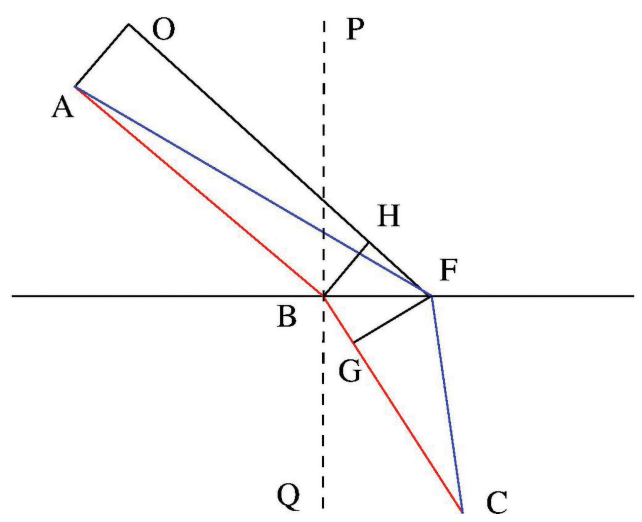

Figure 36: $A B$ and $B C$ are the incident and refracted rays at the point $B$ of an interface BF. AFC is another arbitrary path connecting the points $A$ and $C$. The time to reach point $C$ from $A$ is smaller for the path $A B C$, which obey the sine law, than it is for the path AFC.

Grantham grammar school from 1653 until 1660. He was admitted at the Trinity College, University of Cambridge, in 1661. In 1665, he left to Woolsthorpe because of the great plague but returned to Cambridge two years later, in 1667. He was awarded the degree of Master of Arts soon after his return, and in 1669 was appointed professor of mathematics at Cambridge. In 1696 he accepted the post of warden of the Royal Mint and departed from Cambridge to London. He lived in London for 30 years and died there in 1727 [83.

He wrote on mathematics, optics, mechanics, astronomy, and heat. His theory of light is contained in his books on Optics 84 published in 1704 but in part written much earlier. The distinguishing feature of his theory of light lies in the treatment of color. According to his theory the sun light consists of light rays of different colors having distinct refrangibility. This feature explains the prism experiment that he carried out in 1666 [83] and described in his treatise. A small circular hole is made on the window of a dark chamber through which a sun light beam enters the chamber. The beam passes through a triangular glass prism and is projected on the opposite wall revealing the dispersion of the light in several colors.

His major work Mathematical Principles of Natural Philosophy was published in 1687, in Latin [85. A translation of the third Latin edition appeared in 1729 [86, 87]. He gives a systematic and logical exposition of his theory of motion and of his theory of gravitational attraction. His theory of motion is meant to describe not only terrestrial bodies but also celestial bodies, and his theory of gravitational attraction does not distinguish whether the body is terrestrial or celestial. The treatise comprises two initial sections followed by three books. The first initial section contains the definitions of some quantities, and the second, the statements of the three fundamental laws.

\subsection{Laws of motion}

The definition 1 is that of quantity of matter, or mass, which is defined as arising from its density and bulk conjointly. This is clearly a circular definition which we break by interpreting this statement as the presentation of mass as a primitive concept of the theory. The definition 2 is that of quantity of motion which is defined as arising from the velocity and quantity of matter conjointly, that is, the product of velocity and mass.

The definition 4 is that of impressed force, or force, which is an action exerted upon a body, in order to change its state, either of rest, or uniform in a right line. Again, if we take into account the laws of motion, this is a circular definition. We thus interpret this statement as the presentation of force as a primitive concept. Newton remarks that a force can always be decomposed into two oblique forces according to a parallelogram. In modern terms a force is understood as a vector quantity.

Newton does not give definitions of time and space because, he says, they are well known to all. However, he distinguishes abolute time from relative time and absolute space from relative space. Absolute time flows equably and is synonymous of duration whereas relative time is the sensible measure of duration such as an hour, a day, a month, a year. The absolute space remains similar and immovable whereas relative space is some movable measure of absolute space. The motion on the absolute space is termed absolute motion. A body at rest on a ship on sail is in relative rest but if it moves it will be in relative motion with respect to the ship.

The three postulates or the three fundamental laws of the Principles are:

1. Every body continues in its state of rest, or of uniform motion in a straight line, unless it is compelled to change that state by forces impressed upon it.

2. The change of motion is proportional to motive force impressed; and is made in the direction of the straight line in which that force is impressed. More clearly, the change of the quantity of motion per unit time.

3. To every action there is always opposed an equal reaction: or, the mutual actions of two bodies upon each other are always equal, and directed to contrary parts.

It should be pointed out that velocity and quantity of motion are conceived as comprising direction. In modern terms they are vector quantities. Thus a change in velocity means a change in the speed, or a change in the direction of the motion, or both. Thus the second postulate says that a force is necessary not only to change the speed but also to change the direction of motion, even if the speed remains constant.

The underlying mathematics of the Principles is geometry but Newton combined geometry with calculus, 

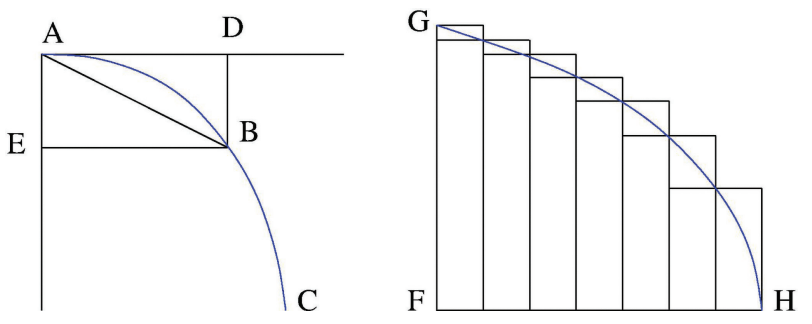

Figure 37: When the point $B$ approaches the point $A$ along the curve $A B C$, the ultimate ratios of the lengths of the arc $A B$, the cord $A B$ and the tangent $A D$ are equal to unity. When the widths of the rectangles decrease and their number increase in infinitum, the sum of the areas of the rectangles approaches the area under the curve $\mathrm{GH}$.

that he invented. This geometric calculus he calls the method of first and last ratios, and is presented in the section 1 of book 1 . The basic rules of his calculus are represented in Figure 37. Consider the point $\mathrm{B}$ on the curve $\mathrm{ABC}$ and the tangent $\mathrm{AD}$, the chord $\mathrm{AB}$, and the $\operatorname{arc} \mathrm{AB}$. When the point $\mathrm{B}$ approaches the point $A$, the ultimate ratios of their lengths are equal to unity. Consider now the area under the curve GH and the areas of the inscribed and circumscribed rectangles When the widths of the rectangles decrease and their number increase in infinitum, their ultimate ratios are equal to unity. In other terms, the sum of the areas of the rectangles approaches the area under the curve.

\subsection{Central forces}

The law of area is presented in section 2 of book 1 . If a body is under the action of a central force then the line joining the body to the center of force sweeps equal areas in equal times. Let us consider the trajectory of a body under the action of a force directed toward the center $\mathrm{S}$, as shown in Figure 38. At equal intervals of time $\tau$, one draws the points $\mathrm{A}, \mathrm{B}, \mathrm{C}, \mathrm{D}$, and $\mathrm{E}$ on the curve. The continuous trajectory is replaced by the sequence of straight line segments. Along each line segment the velocity of the body is constant and its length is $\tau$ multiplied by the velocity. The change in velocity at the point $\mathrm{B}$ is obtained by drawing the parallelogram ABCK. The line segment BK gives the change in velocity multiplied by $\tau$, and by the second postulate, it must have the direction $\mathrm{BS}$ of the impressed force. We thus conclude that $\mathrm{K}$ is on the line $\mathrm{BS}$.

The triangles SBA and SBC have the same area because they have a common base $\mathrm{SB}$ and their heights are equal because, as ABCK is a parallelogram, the triangles KBA and $\mathrm{KBC}$ have equal heights. By similar reasonings, the triangles $\mathrm{SAB}, \mathrm{SBC}, \mathrm{SCD}$, and SDE have equal areas. To complete the demonstration it suffices to decrease the lengths of the segments and increase their number in infinitum so as to approach the original trajectory.

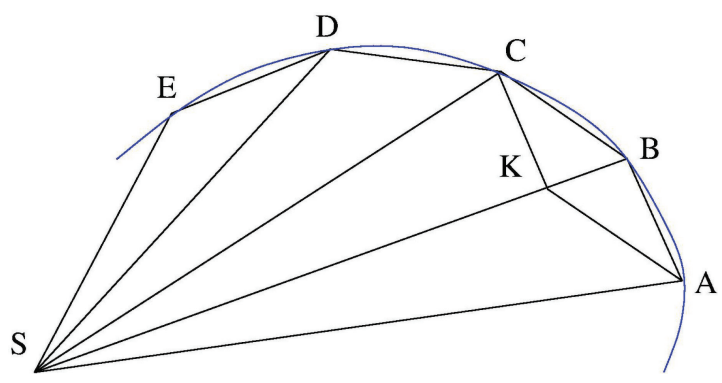

Figure 38: The trajectory of a body under the action of a central force toward the center $S$ is approximated by a polygonal path. The points $A, B, C, D$, and $E$ are chosen so that the intervals of time for traveling each line segment are the same. ABCK is a parallelogram and $\mathrm{K}$ is on the line $\mathrm{SB}$.

The demonstration that centripetal force is proportional to the square of the velocity divided by the radius of the circular path is carried out by considering that the curve of Figure 38 is a circumference. In this case, the triangles $\mathrm{SBC}$ and $\mathrm{KBE}$ become similar isosceles triangles from which follows that $\mathrm{SB} / \mathrm{BC}=\mathrm{BC} / \mathrm{KB}$. Denoting by $\tau$ the interval of time, by $r$ the radius of the circumference, by $v$ the velocity, then SB equals $r$, $\mathrm{BC}$ equals $\tau v$, and $\mathrm{KB}=\tau^{2} v^{2} / r$. As $\mathrm{KB}$ equals $\tau$ times the change in velocity, this quantity is $\tau v^{2} / r$. From the second postulate, the force $F$ is the mass $m$ times the change in velocity per unit time, or

$$
F=m \frac{v^{2}}{r} .
$$

Let us suppose that the force acting on the body in circular motion is inversely proportional to the square of the radius. Then, using the formula (34), we see that $v$ will be proportional to $r^{-1 / 2}$ and as $v$ is the perimeter of the circumference divided by the period $T$ it follows that $T$ is proportional to $r^{3 / 2}$.

The law of areas allows us to formulate the second fundamental law in geometric terms alone. Let $\mathrm{P}$ and $\mathrm{Q}$ two points of a trajectory separated in time by an interval of time $\tau$. Let $\mathrm{RP}$ be a tangent to $\mathrm{P}$ and $\mathrm{QV}$ a parallel to the tangent at the point $\mathrm{Q}$, as shown in Figure 39 The line $\mathrm{PC}$ connects the point $\mathrm{P}$ to the center of force C, and QR and QT are, respectively, parallel and perpendicular to $\mathrm{PC}$. The line segment $\mathrm{RP}$ is the velocity of the body multiplied by $\tau$ and $\mathrm{QR}$ is the change in velocity multiplied by $\tau$. As, by the second postulate, the force is proportional to the change in velocity divided by $\tau$, then it is proportional $\mathrm{QR} / \tau^{2}$. Using the law of areas, we see that the area of the triangle CQP, which is CP.QT, is proportional to $\tau$. This result allows us to replace $\tau$ by the area and reach the result that the force is proportional to

$$
\frac{\mathrm{QR}}{\mathrm{CP}^{2} \cdot \mathrm{QT}^{2}} \text {. }
$$

If a body revolves in an ellipse, the law of force attracting the body to its center is proportional to 


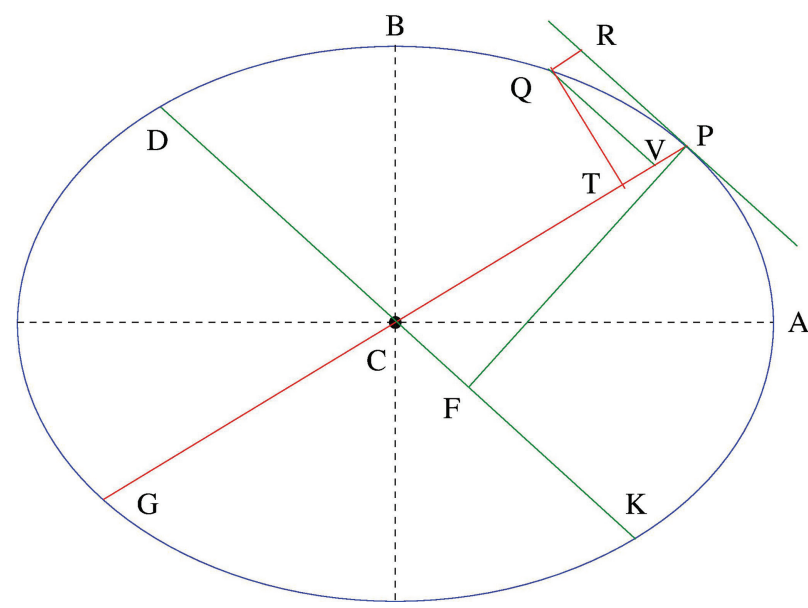

Figure 39: $A$ body $P$ describes the ellipse $A B G K$ and is under an attractive central force directed towards center $C$ of the ellipse. The line RP is tangent to the ellipse at $P$ and $Q V$ and the diameter DCK are parallel to RP, and PF is perpendicular to $R P$. The line $Q R$ is parallel to the line $C P$ joining $P$ to the center $\mathrm{C}$ of the ellipse and $\mathrm{QT}$ is perpendicular to $\mathrm{CP}$.

distance to its center. This proposition is demonstrated by using the result 35 and two geometric properties of an ellipse. If $\mathrm{V}$ is a point on $\mathrm{CP}$, and $\mathrm{DC}$ is parallel to the tangent at $\mathrm{P}$, then the first property is

$$
\frac{\mathrm{GV} \cdot \mathrm{PV}}{\mathrm{QV}^{2}}=\frac{\mathrm{CP}^{2}}{\mathrm{CD}^{2}}
$$

If we let $\mathrm{PF}$ be perpendicular to $\mathrm{DC}$, then the second property of the ellipse is

$$
\mathrm{BC} \cdot \mathrm{CA}=\mathrm{CD} \cdot \mathrm{PF} .
$$

Considering that the rectangular triangles $\mathrm{PFC}$ and $\mathrm{QTV}$ are similar then $\mathrm{QV} / \mathrm{QT}=\mathrm{CP} / \mathrm{PF}$ or, using the two properties of the ellipse, $\mathrm{PV} / \mathrm{QT}^{2}=\mathrm{PC}^{4} / a^{2} b^{2} \cdot \mathrm{GV}$ where $a$ and $b$ are the semi-major and semi-manor axis of the ellipse, $\mathrm{CA}$ and $\mathrm{CB}$. When the point $\mathrm{Q}$ approaches $\mathrm{P}, \mathrm{GV}$ approaches $2 \mathrm{PC}$ and $\mathrm{PV} / \mathrm{PC}^{2} \cdot \mathrm{QT}^{2}=\mathrm{PC} / 2 a^{2} b^{2}$. Recalling that $\mathrm{PV}$ equals $\mathrm{QR}$ we see that the force is proportional to $\mathrm{PC}$, the distance of the body to the center of the ellipse.

\subsection{Motion in eccentric conic section}

In section 3 of book 1, Newton poses the problem of finding the law of force concerning the motion of a body on trajectory which is a conic section, an ellipse, a parabola, or a hyperbola. Newton shows that the force is inversely proportional to the square of the distance of the body to center of force located at the focus of the conic section. For the elliptical trajectory the demonstration is as follows [88]. Let $\mathrm{RP}$ be a tangent to a point $\mathrm{P}$ of an ellipse, $\mathrm{QV}$ a parallel to the tangent that crosses the ellipse at the point Q, and DC another parallel to the tangent at the center $\mathrm{C}$ of the ellipse, as shown in

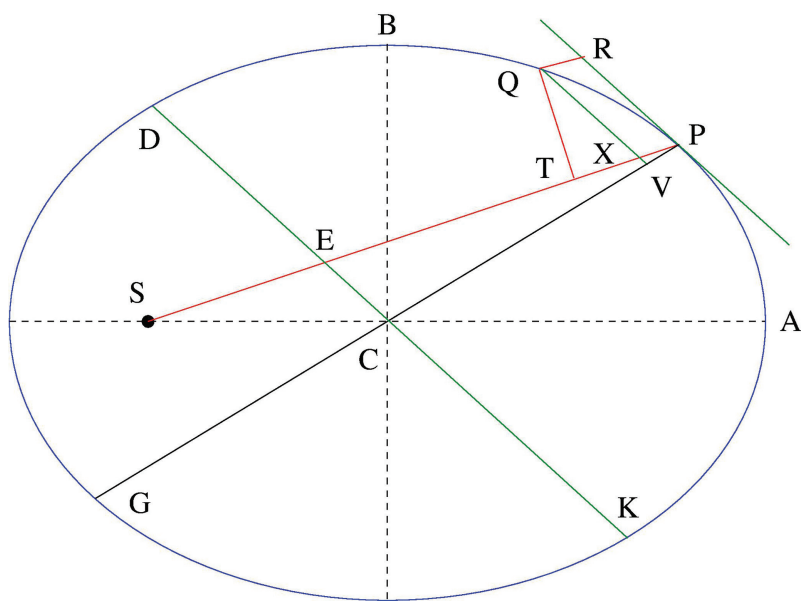

Figure 40: $A$ body $P$ describes the ellipse $A B G K$ and is under an attractive central force directed towards the focus $S$ of the ellipse. The line RP is tangent to the ellipse at $P$ and $Q V$ and the diameter DCK are parallel to RP. The line $\mathrm{QR}$ is parallel to the line CS joining $P$ to the focus $S$ of the ellipse and QT is perpendicular to $\mathrm{CS}$.

Figure 40 The line SP connects the focus $\mathrm{S}$ to the point $\mathrm{P}, \mathrm{QR}$ is a parallel to $\mathrm{SP}$, and $\mathrm{QT}$ is perpendicular to $\mathrm{SP}$. The line CP connects the center of the ellipse to the point $\mathrm{P}$.

The demonstration uses the result (35) but in the present case the center of force is the focus $\mathrm{S}$ and the force acting on the body at $\mathrm{P}$ is proportional to

$$
\frac{\mathrm{QR}}{\mathrm{SP}^{2} \cdot \mathrm{QT}^{2}} \text {. }
$$

Considering that $\mathrm{XV}$ and $\mathrm{EC}$ are parallel, then $\mathrm{XP} / \mathrm{PV}=\mathrm{PE} / \mathrm{PC}$ or $\mathrm{QR} / \mathrm{PV}=\mathrm{AC} / \mathrm{CP}$, where we used a third property of an ellipse $\mathrm{PE}=\mathrm{AC}$, and that $\mathrm{XP}=\mathrm{QR}$. Using the first property of an ellipse mentioned above, we find $\mathrm{QR} \cdot \mathrm{GV} / \mathrm{QV}=\mathrm{CP} \cdot \mathrm{AC} / \mathrm{CD}^{2}$. When $\mathrm{Q}$ and $\mathrm{P}$ coincide, we may replace $\mathrm{GV}=\mathrm{GP}=2 \mathrm{AC}$ to find

$$
\frac{\mathrm{QR}}{\mathrm{QV}^{2}}=\frac{\mathrm{AC}}{2 \mathrm{CD}^{2}} \text {. }
$$

Taking into account that $\mathrm{QT}$ is perpendicular to $\mathrm{PE}$ and $\mathrm{PF}$ is perpendicular to $\mathrm{EP}$, then $\mathrm{QX} / \mathrm{QT}=\mathrm{PE} / \mathrm{PF}$ or $\mathrm{QX} / \mathrm{QT}=\mathrm{CA} / \mathrm{PF}$. Using the second property of an ellipse mentioned above, we find

$$
\frac{\mathrm{QX}}{\mathrm{QT}}=\frac{\mathrm{CD}}{C B} \text {. }
$$

Multiplying equation (39) by the square of (40), and taking into account that QX approaches QV when the point $\mathrm{Q}$ approaches $\mathrm{P}$, we reach the result

$$
\frac{\mathrm{QR}}{\mathrm{QT}^{2}}=\frac{a}{2 b^{2}} \text {. }
$$

Replacing this result in equation $(38)$ and recalling that $\mathrm{SP}$ is the distance of the body to the focus $\mathrm{S}$ of the ellipse, we reach the desired result, namely that the force is inversely proportional to the square of the distance. 


\subsection{Attractive forces of spherical bodies}

Up to section 11 of book 1, Newton treated the motion and forces between corpuscles understood as bodies with sizes small when compared with the distances between them. In the following section, he starts by considering bodies that are not small compared to their distance, particularly spherically symmetric bodies. In section 12 of book 1, he analyzes the force acted on a corpuscle by a spherical surface assuming that the force between the corpuscle and any element of the spherical surface is inversely proportional to the square of the distance. The mass is uniformly distributed on the spherical surface so that the mass of a portion of the surface is proportional to its area.

If the corpuscle is located inside the spherical surface, the total force on the corpuscle vanishes. This result is shown by considering two opposite elements of the surface in relation to the corpuscle. The ratio of the areas and thus ratio of their masses will be equal to the ratio of the square of their distances to the corpuscle. As the force on the corpuscle is proportional to the mass of the element and inversely proportional to the square of the distance, it follows that the forces are equal and opposite.

If the corpuscle is located outside the spherical surface, the total force on the corpuscle will be inversely proportional to the square of the distance of the corpuscle to the center of the sphere. That is, the force of attraction is the one that results if all the mass were concentrated at the center. This crucial theorem is stated as proposition 71 and its geometric demonstration presented in the Principles has been considered to be difficult and "must have left its readers in helpless wonder" 89. We will not try to reproduce the demonstration given by Newton and instead present another geometric demonstration.

The corpuscle is located at $\mathrm{P}$ and $\mathrm{PE}$ and $\mathrm{PF}$ are straight lines that cut the spherical surface as shown is Figure 41. The force at $\mathrm{P}$ due to the element EF is proportional to $1 / \mathrm{PE}^{2}$. The projection of force along the direction $\mathrm{PS}$ will be proportional $(\mathrm{PC} / \mathrm{PE})\left(1 / \mathrm{PE}^{2}\right)$. If we take into account the ring generated by the segment $\mathrm{EF}$ as the semicircle AEFB revolves around the diameter $\mathrm{AB}$, the force along PS will be proportional to the area of the ring and thus proportional to

$$
\frac{\mathrm{EF} \cdot \mathrm{EC} \cdot \mathrm{PC}}{\mathrm{PE}^{3}} \text {. }
$$

Now let us consider the difference

$$
\frac{\mathrm{FS}^{3}-\mathrm{FS} \cdot \mathrm{DS} \cdot \mathrm{PS}}{\mathrm{PS}^{2} \cdot \mathrm{PF}}-\frac{\mathrm{ES}^{3}-\mathrm{ES} \cdot \mathrm{CS} \cdot \mathrm{PS}}{\mathrm{PS}^{2} \cdot \mathrm{PE}} .
$$

If $\mathrm{EF}$ is small enough the expressions 42 and 43 becomes equal or in the language used by Newton, the ratio between them becomes equal to one when $\mathrm{F}$ approaches E. This is carried out by taking into account that $\mathrm{PF}^{2}=\mathrm{PE}^{2}+2(\mathrm{ES}+\mathrm{FS}) \cdot \mathrm{CD}$, and that the ratio $\mathrm{CD} / \mathrm{EF}$ equals $\mathrm{EC} / \mathrm{ES}$ when the point $\mathrm{F}$ approaches $\mathrm{E}$.

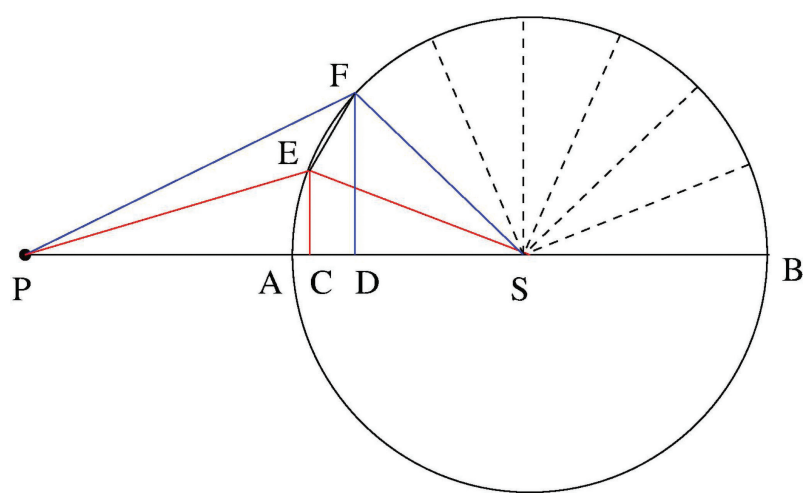

Figure 41: $A E F B$ is a cross section of a spherical surface with center at $\mathrm{S}$. The mass is distributed uniformly on the surface. Each element of the surface attracts a corpuscle placed at the point $\mathrm{P}$ with a force inversely proportional to the square of the distance. The lines PE and PF join the ends of the element EF to the point $P$, and $E C$ and $F D$ are perpendicular to $P S$.

To find the total force acting on the point $\mathrm{P}$, we have to sum all terms of the type (42), or equivalently, of the type (43), along the semicircle AEFB. The result is

$$
\frac{R^{3}-R r^{2}}{r^{2}(r+R)}-\frac{R^{3}-R r^{2}}{r^{2}(r-R)}=\frac{2 R^{2}}{r^{2}},
$$

where $R$ is the radius of the spherical surface and $r$ is the distance PS of the corpuscle to the center of the sphere.

Newton generalizes the above results to a spherical body by considering it as made up by concentric spherical surfaces. If the corpuscle outside the sphere it will be attracted with a force inversely proportional of the distance to the center of the sphere. If the corpuscle is inside the sphere and if the sphere is homogeneous, then the force towards the center will be proportional to the distance to the center.

\subsection{Gravitation}

In the book 3 of the Principles, Newton presents the law of gravitation in the form of a statement concerning spherical bodies with a distribution of mass which depends only on the distance from its center. The force of gravitational attraction between two such bodies are inversely proportional to the square of the distance from their center and proportional to their masses.

Instead of spherical bodies, the gravitational law could be stated more generically in terms of particles. But in this case, we face the problem of deriving the inverse square laws for spherical bodies. The derivation was given by Newton in book 1 , as we have seen above. This solution of this problem appears to be the cause of the delay in the announcing the law of gravitation and the publication of the Principles as he already had conceived the law in 1666 [87].

As the acceleration is the force divided by the mass, the acceleration of a body due to the gravitational attraction is independent of its mass. In accordance with 


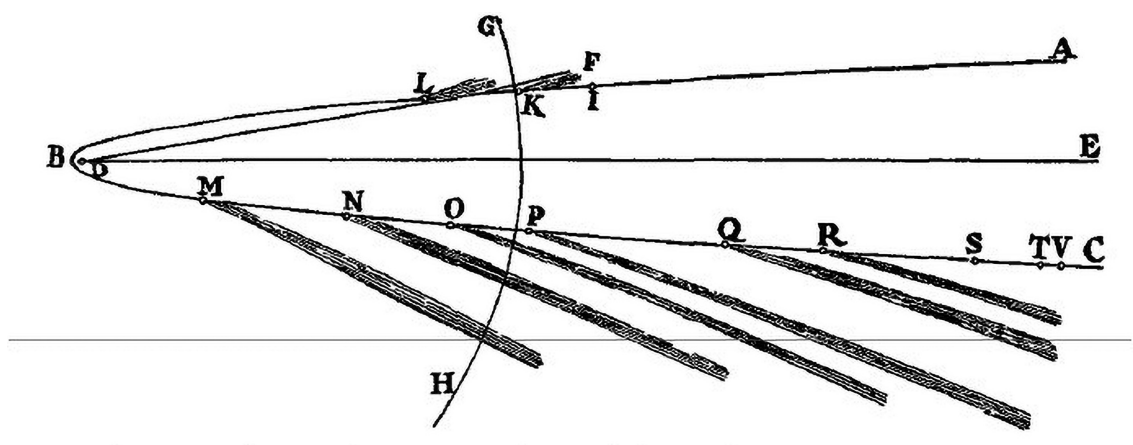

Figure 42: Orbit of the great comet of 1680 taken from an illustration of the Principles [87]. ABC represents the orbit of the comet, D the sun, GH the intersection with the sphere of the orbit of the earth. The points I, K, L, M, N, O, P, Q, R, S, T, and $\mathrm{V}$ represent the place of the comet at November 4, 11, 19, December 12, 21, 29, 1680, January 5, 25, February 5, 25, March 5, 9, 1681 , respectively.

the law of gravitation the acceleration at a certain point due to a spherical body is inversely proportional to the distance to the center of the sphere. This result allowed Newton to determine the acceleration at the surface of the earth from that of the moon. Newton assumed the distance of the moon from the earth to be 60 semidiameters of the earth and the orbital period to be 27 days, 7 hours, and 43 minutes and the circumference of the earth to be 123249600 Paris feet. From these results, he determined the acceleration of the moon, and dividing it by $60 \times 60$ he obtained the acceleration at the surface of the earth. He presented the results in terms of the space traveled by a body in one second, which is half the acceleration. The value he found was 15 feet, 1 inch, and 1 line 4/9, which he compared with the value 15 feet, 1 inch, and 1 line 7/9, obtained by Huygens from the oscillations of a pendulum. Newton concluded that the force by which the moon is retained in its orbit is that very same force which we commonly call gravity.

Assuming the inverse square laws for spherical bodies, and taking into account the results of book 1 derived from this fundamental law, Newton could announce several laws concerning the motion of celestial bodies. The planets move in ellipses which have their common focus in the center of the sun. By radii drawn to that center, they describe areas proportional to the time of description. The principal diameter of the ellipses is proportional to the power $2 / 3$ of the periodic time.

Newton also considers the motion of the comets remarking that their orbits are a conic section usually an elongated ellipse or a parabola. As an example he examines the orbit of the great comet of 1680 , observed by Kirch on November 4 of that year. From the observations of Flamsteed and from his own, Newton could fit a parabola to the positions of the comet. The result is shown in the illustration of Figure 42

Newton observes that comets with great bulk and splendor tails may have obscure and small heads. He then mentions the comet seen in Brazil, March 5, 1668,
7 pm, by Valentin Estancel. The comet appeared "near the horizon, and toward the southwest, with a head so small as scarcely to be discerned, but with a tail above measure splendid, so that the reflection thereof from the sea was easily seen by those who stood on the shore; it looked like a fiery beam extended 23 degrees in length from the west to south, almost parallel to the horizon." Estancel was the Czech jesuit astronomer who was sent to Brazil where he made the comet observation in Salvador. His original name was Stansel 90.

As a final section of the second and third Latin edition of the Principles, Newton added an essay where he discusses the causes of gravity. In the very beginning of the essay, Newton counters the hypothesis of vortices stating that it could not explain the very eccentric orbits of comets. He then explains that his theory of action at distance through the empty space is not unreal arguing that falling bodies with distinct weights descent in the void with the same velocity by the action of gravity. Thus the celestial bodies remains in their orbits in empty space also by the action of gravity.

He says that he was not able to find the causes of gravity from the phenomena and for that reason he framed no hypotheses. Indeed, the whole book 3 of the Principles, containing the theory of gravitation, the inverse square law is not presented as a general hypothesis or as an axiom but as derived from the phenomena. For instance, the theorem 2, stating that the planets are attracted to the sun according to the inverse square law, is derived from the areal law and the three-half law, presented by Newton as phenomena.

It seems that Newton distinguishes fundamental laws that are causes from those that are not. Thus the three fundamental laws of motion are understood as the causes of motion, particularly the second law. But, for Newton, the inverse square law is not the cause of gravitation and for this reason, the law must be derived or more properly, it should be inferred from the phenomena and rendered general by induction, as he puts it. 
Table 1: The author of a scientific theory is listed together with the abbreviated name of the main work where it is found and the year of its publication, or the century (in Roman numeral) of its conception. The last column gives the main elementary concepts of the theory.

\begin{tabular}{lllll}
\hline Author & \multicolumn{1}{c}{ Work } & Year & Scientific theory & Elementary concepts \\
\hline Euclid & Elements & III BC & geometry & space \\
Aristarchus & Distances of the Sun and Moon & III BC & astronomy & space \\
Archimedes & Equilibrium of Planes & III BC & statics & space, weight \\
Archimedes & Floating Bodies & III BC & hydrostatics & space, weight \\
Ptolemy & Almagest & II & astronomy & space, time \\
Ibn al-Haytham & Book of Optics & XI & optics & light ray \\
Copernicus & Revolutions of the Heavenly Bodies & 1543 & astronomy & space, time \\
Kepler & New Astronomy & 1609 & astronomy & space, time \\
Galileo & Two New Sciences & 1638 & mechanics & space, time, weight \\
Descartes & Discourse on the Method & 1637 & optics & light ray \\
Pascal & Equilibrium of Liquids & 1663 & fluid statics & pressure \\
Huygens & Pendulum Clock & 1673 & mechanics & space, time, force \\
Huygens & Treatise on Light & 1690 & optics & light wave \\
Newton & Mathematical Principles & 1687 & mechanics & space, time, mass, force \\
\hline
\end{tabular}

\section{Conclusion}

The structure of a scientific theory was shown to consist of an hierarchy of three levels. The highest level is composed by rational thinking understood as an innate ability that emerges around the end of childhood and beginning of adolescence. Rational thinking organizes the second level which is that of the symbolic framework or the abstract part of the scientific theory. The rules imposed by rational thinking demands that the scientific theory should be composed of primitive concepts and postulates or fundamental laws from which other concepts and laws are derived by deductive reasoning. The symbolic framework is a representation of the real world or the phenomena, which constitutes the third level of the structure.

We point out that rational thinking which is innate should not be confused with the formal logic, or symbolic logic, which was systematized by Boole [91, and was based on the logic of Aristotle, which is a theory. Nonetheless, the rules of symbolic logic parallels those of rational thinking and those that follows from it.

Based on the structure of scientific theory, we have analyzed several physical theories that emerged along the course of time since the Hellenistic period up to the seventeen century. They are listed in Table 1 together with their authors, the time of their conception or publication, and the main primitive or elementary concepts. We recall that these concepts are the ones that are undefined. Most of the physical theories have space and time as their primitive concepts. It is a common feature of these theories that the underlying mathematics is geometry. From the beginning of the eighteenth century, the differential and integral calculus became the new mathematical tool in physical sciences allowing a great development of the theories, which is also worth of a detailed analysis in the light of the point of view regarding scientific theories presented here.

\section{Acknowledgements}

I wish to acknowledge Tânia Tomé for her critical reading of the manuscript, Vera B. Henriques for conversations on the cognitive development of children, Francisco A. de Oliveira for calling my attention to Pascal's thoughts, and Luís G. de Oliveira for discussions about the ideas of the ancient Greeks.

\section{References}

[1] E. Nagel, The Structure of Science (Harcourt Brace and World, New York, 1961).

[2] C.G. Hempel, Aspects of Scientifc Explanations (Free Press, New York, 1965).

[3] W.C. Salmon, The Foundations of Scientific Inference (University of Pittsburgh Press, Pittsburgh, 1966).

[4] R. Carnap, An Introduction to the Philosophy of Science (Basic Books, New York, 1966).

[5] L.C. Menezes, A Matéria, Uma Aventura do Espírito (Editora Livraria da Física, São Paulo, 2005).

[6] R. Dugas, A History of Mechanics (Routledge and Kegan Paul, Lodon, 1955).

[7] T.S. Kuhn, The Structure of Scientific Revolutions (University of Chicago Press, Chicago, 1962).

[8] E. Segrè, From Falling Bodies to Radio Waves (Freeman, New York, 1984).

[9] L. Russo, The Forgotten Revolution (Springer, Berlin, 2004).

[10] A.S.T. Pires, Evolução das Idéias da Fisica (Editora Livraria da Física, São Paulo, 2011), 2 ed.

[11] L.R. Evangelista, Perspectivas em História da Física, Dos Babilônios à Sintese Newtoniana (Editora Ciência Moderna, São Paulo, 2011). 
[12] L.R. Evangelista, Perspectivas em História da Física, Da Física dos Gases à Mecânica Estatística (Editora Livraria da Física, São Paulo, 2014).

[13] L.Q. Amaral, Revista de História da Ciência 5, 89 (2018).

[14] B. Pascal, Pensées (Desprez, Paris, 1669).

[15] R. Descartes, Discours de la Methode, la Dioptrique, les Meteores et la Geometrie (Maire, Leyde, 1637).

[16] D. Johanson and B. Edgar, From Lucy to Language (Simon and Schuster, New York, 1996).

[17] J. Piaget, Genetic Epistemology (Norton, New York, 1971).

[18] B.J. Wadsworth, Piaget's Theory of Cognitive and Affective Development (Longman, London, 1989), 4 ed.

[19] K.S. Berger, The Developing Person through the Life Span (Worth, New York, 2008), 7 ed.

[20] L.C. Gomes and L.M. Bellini, Rev. Bras. Ens. Fis. 31, 2301 (2009).

[21] K. Craik, The Nature of Explanation (Cambridge University Press, Cambridge, 1943).

[22] G.H. Luquet, Le Dessin Enfantin (Alcan, Paris, 1927).

[23] M.L.B. Duarte, in Anais do XVI Encontro Nacional da ANPAP (Florianópolis, 2007).

[24] P. Duhem, La Théorie Physique, Son Objet et da Structure (Chevalier et Rivière, Paris, 1906).

[25] J.R.N. Chiappin, Transversal International Journal for the Historiography of Science 2, 36 (2017).

[26] C.C. Laranjeiras, J.L. da Silva and J.R.N. Chiappin, Rev. Bras. Ens. de Fis. 39, e4602 (2017).

[27] N.M.M. Kiouranis, A.R. de Souza and O. Santin Filho, Rev. Bras. Ens. Fis. 32, 1507 (2010).

[28] S. Drake, Galileo at Work (University of Chicago Press, Chicago, 1978).

[29] N.J. Nersessian, in Proceedings of the 1992 Biennial Meeting of the Philosophy of Science Association (Michigan, 1992).

[30] J.E. Ruby, J. Hist. Ideas 47, 341 (1986).

[31] G. Sarton, A history of Science, Hellenistic Science and Culture in the Last Three Century B. C. (Harvard University Press, Cambridge, 1959).

[32] Euclid, The Thirteen Books of Euclid's Elements (Cambridge University Press, Cambridge, 1908).

[33] H. Eves, An Introduction to the History of Mathematics (Holt, Rinehart and Winston, New York, 1969).

[34] T.L. Heath, Aristarchus of Samos (Clarendon Press, Oxford, 1913).

[35] T.B. de Oliveira, V.T. Lima and A.C. Bertuola, Rev Bras. Ens. Fis. 38, e2304 (2016).

[36] T.L. Heat (ed), The Works of Archimedes (Cambridge University Press, Cambridge, 1897).

[37] E.J. Dijksterhuis, Archimedes (Ejnar Munksgaard, Copenhagen, 1956).

[38] M.J. Crowe, Theories of the World from Antiquity to the Copernican Revolution (Dover, New York, 1990).

[39] G.J. Toomer, Ptolemy's Almagest (Duckworth, London, 1984).

[40] R. Rashed, Geometry and Dioptrics in Classical Islam (Al-Furkān, London, 2005).

[41] A. Mark Smith, Transaction of the American Philosophical Society 91, 1 (2001).
[42] A. Mark Smith, Transaction of the American Philosophical Society 96, 1 (2006).

[43] A. Mark Smith, Transaction of the American Philosophical Society 98, 1 (2008).

[44] A. Mark Smith, Transaction of the American Philosophical Society 100, 1 (2010).

[45] A. Mark Smith, Transaction of the American Philosophical Society 86, 1 (1996).

[46] R. Goulding, Arch. Hist. Exact. Sci. 72, 497 (2018).

[47] A. Kwan, J. Dudley and E. Lantz, Phys. World 15, 64 (2002).

[48] R. Rashed, ISIS 81, 464 (1990).

[49] J.L.E. Dreyer, A History of Astronomy from Thales to Kepler (Dover, New York, 1953).

[50] N.M. Swerdlow and O. Neugebauer, Mathematical Astronomy in Copernicus's De Revolutionibus (Springer, New York, 1984).

[51] E. Rosen, Copernicus On the Revolutions (Polish Scientific Publishers, Poland, 1978).

[52] E. Rosen, Three Copernican Treatises (Dover, New York, 1959), 2 ed.

[53] M. Caspar, Kepler (Dover, New York, 1993).

[54] J. Kepler, Astronomia Nova, (1609).

[55] W.H. Donaue, Johannes Kepler New Astronomy (Cambridge University Press, Cambridge, 1992).

[56] J. Kepler, Harmonices Mundi (Plancus, Lincii, 1619).

[57] C. Wilson, Isis 59, 4 (1968).

[58] M. Sharratt, Galileo Decisive Innovator (Cambridge University Press, Cambridge, 1994).

[59] G. Galilei, Sidereus Nuncius (Baglionum, Venetiis, 1610).

[60] E.S. Carlos, The Sidereal Messenger of Galileo Galilei (Rivingtons, London, 1880).

[61] J.M. Pasachoff, J. Hist. Astr. 46, 218 (2015)

[62] G. Galilei, Il Saggiatore (Mascardi, Roma, 1623).

[63] G. Galilei, Dialogo sopra i Due Massimi Sistemi del Mondo (Landini, Fiorenza, 1632).

[64] G. Galilei, Discorsi e Dimostrazioni Matematiche intorno à Due Nuove Scienze (Elsevirii, Leida, 1638).

[65] G. Gallilei, Dialogues Concerning Two New Sciences (MacMillan, New York, 1914).

[66] C. Huygens, Horologium Oscillatorium (Muguet, Paris, 1673).

[67] C. Huygens, Oeuvres Complètes (Nijhoff, La Haye, 1935), tome 18 .

[68] J.F. Scott, The Scientific Work of René Descartes (Taylor and Francis, London, 1952).

[69] D.M. Clarke, Descartes: a Biography (Cambridge University Press, Cambridge, 2006).

[70] R. Descartes, Discourse on Method, Optics, Geometry, and Meteorology (Bobbs-Merrill, Indianopolis, 1965).

[71] J. Mesnard, Pascal: His Life and Works (Philosophical Library, New York, 1952).

[72] F. Strowski, Pascal, Oeuvres Complètes (Ollendorff, Paris, 1923), v. 1.

[73] B. Pascal, Expériences Nouvelles Touchant le Vide (Margat, Paris, 1647)

[74] E. Torricelli, Lezioni Accademiche (Guiducci e Franchi, Firenze, 1715). 
[75] M. Pascal, Récit de la Grande Expérience de l'Équilibre des Liqueurs (Saureux, Paris, 1648).

[76] B. Pascal, Traités de l'Équilibre de Liqueurs et de la Pesanteur de la Masse de l'Air (Desprez, Paris, 1663).

[77] A.E. Bell, Christian Huygens (Arnold, London, 1947).

[78] A.I. Sabra, Theories of Light, From Descartes to Newton (Cambridge University Press, Cambridge, 1981).

[79] C. Huygens, Traité de la Lumière (Pieter van der Aa, Leide, 1690)

[80] C. Huygens, Treatise on Light (Macmillan, London, 1912).

[81] P.M.C. Dias, Rev. Bras. Ens. Fis. 35, 1602 (2013).

[82] M. Burrowes and C. Farina, Rev. Bras. Ens. Fis. 27, 175 (2005).

[83] A.R. Hall, Isaac Newton Adventurer in Thought (Blackwell, Oxford, 1992).

[84] I. Newton, Opticks (Smith and Walford, London, 1704).

[85] I. Newton, Philosophiae Naturalis Principia Mathematica (Streater, Londini, 1687).

[86] I. Newton, Mathematical Principles of Natural Philosophy (Motte, London, 1729).

[87] F. Cajori, Sir Isaac Newton's Mathematical Principles (University of California Press, Berkeley, 1974), 2 v.

[88] C.M. Porto, Rev. Bras. Ens. Fis. 37, 1602 (2015).

[89] J.E. Littlewood, The Mathematical Gazette 32, 179 (1948).

[90] V. Estancel, Il Giornale de Letterati per tutto l'anno 1673 (Nicolò Angelo Tinassi, Roma, 1673).

[91] G. Boole, An Investigation of the Laws of Thought (Walton and Maberly, London, 1854). 